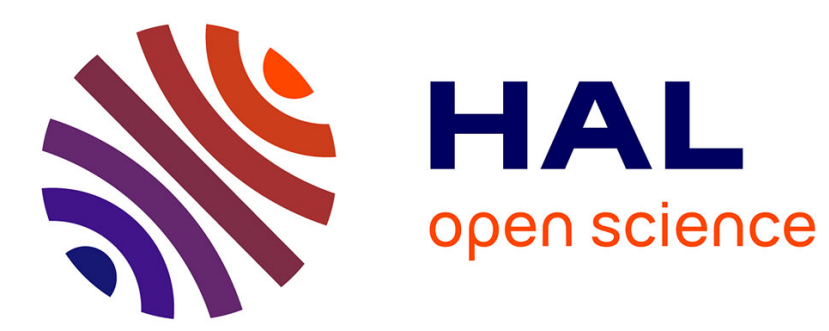

\title{
Interfaces of Incompleteness
}

Giuseppe Longo

\section{To cite this version:}

Giuseppe Longo. Interfaces of Incompleteness. Minati, Gianfranco; Abram, Mario R.; Pessa, Eliano. Systemics of Incompleteness and Quasi-Systems, Springer, 2018, 3030152766, 978-3030152765. hal02903234

\section{HAL Id: hal-02903234 \\ https://hal.science/hal-02903234}

Submitted on 21 Jul 2020

HAL is a multi-disciplinary open access archive for the deposit and dissemination of scientific research documents, whether they are published or not. The documents may come from teaching and research institutions in France or abroad, or from public or private research centers.
L'archive ouverte pluridisciplinaire HAL, est destinée au dépôt et à la diffusion de documents scientifiques de niveau recherche, publiés ou non, émanant des établissements d'enseignement et de recherche français ou étrangers, des laboratoires publics ou privés.

\section{(이) $\$$}

Distributed under a Creative Commons Attribution - NonCommercial - NoDerivatives| 4.0 
In Minati, G, Abram, M \& Pessa, E (Eds.) Systemics of Incom-

pleteness and Quasi-systems, Springer, New York, NY, 2018

\section{Interfaces of Incompleteness}

\section{Giuseppe Longo}

Centre Cavailles, République des Savoirs, CNRS

College de France et Ecole Normale Supérieure

and School of Medicine, Tufts University, Boston

http://www.di.ens.fr/users/longo

Introduction

1. From Laplace to Poincaré

2. From Geometry to Logic

3. From Hilbert to Gödel

3.1. ... Through Poincaré and Weyl

3.2. Arithmetics, an Absolute

4. The Theorem

4.1. And What About "truth"?

5. Poincaré vs. Gödel

5.1. Turing: From Formal Systems to Continuous Dynamics

6. Einstein and the Thesis of the Incompleteness of Quantum Mechanics

7. The Mathematical Incompleteness of Formal Theories

7.1. Towards the Cognitive Foundations of Induction

8. Information and Encoding in the Cell

References

A preliminary version of this paper appeared as "Incompletezza", in C. Bartocci and P. Odifreddi (Eds.), La Matematica, vol. 4, (pp. 219-262), Einaudi, Torino, 2010. 


\section{Introduction}

In addition to being one of the main results in Mathematical Logic, Gödel's 1931 Incompleteness Theorem can also serve as a starting point for a reflection extending beyond Mathematics and the issue of its foundations in order to relate them to problems and methods pertaining to other disciplines. It is in the light of Gödel's theorem that we will present a "critical history of ideas", that is, an explicitly a posteriori reading of some marking points of modern scientific thought, times when the audacity of the propositions of knowledge would be tempered by problems demonstrated to be unsolvable and by negative or limitating results. Negative results, however, opened up new horizons for knowledge. We will examine some of the main scientific paradigms in order to find, within their respective domains, their common thread, that is, incompleteness, understood in its various meanings.

The detailed analysis, although informal, of Gödel's theorem and a reflection on Turing's work will constitute but one element of this text. Thus, we will first see the way in which incompleteness and limits of well-established approaches have been demonstrated and sometimes surpassed, starting with Poincaré's fundamental work. This will allow to broaden our reading grid - while avoiding, we hope, inappropriate abuses and contaminations - to Laplace's scientific and epistemological approach and to the limits set by Poincaré's major "negative result", as he called it himself.

After discussing Gödel's incompleteness, we will continue with Einstein's theses on the non-"completeness" of Quantum Mechanics, to use the term employed in the very famous article written in collaboration with Podolski and Rosen which analyses this notion. Biology has been dramatically affected by the myth of completeness of molecular descriptions, both in ontogenesis and phylogenesis: DNA as a program and "blue print" of the organism. The richness of organismal individuation and the intrinsic unpredictability of phylogenetic dynamics is then lost as well as the open ended changes of the pertinent phase space of biological evolution. Jointly to an appreciation of the role of rare events in evolution, some understanding of the limits of knowledge set the grounds for new approaches and may help to focus on more suitable a priori for the science of life.

\section{From Laplace to Poincaré}

As regards Laplace (1749-1827), what one must look for is the unity of the method (and of the Universe), and hence the identity between physical laws at our scale of perception and the laws which govern microscopic particles. All observable phenomena are reducible to the elementary ontology underlying matter, movement and force. And at this level, any analysis must base itself on the possibility of isolating, mathematically, a single elementary particle and of describing its movements. It must then, by means of mathematical integration operations, reconstruct the expression of the law of interaction at a distance in particle systems. The analysis 
of planetary systems must thus advance by progressive composition of individual movements and lead to an understanding of the "system" as the sum of the individual behaviors and their interactions, two by two, three by three ...

This mechanistic reduction is intimately linked, for Laplace, to the structure of the determination of all physical events. For all the great physicists of the XVIIIth and $X I X^{\text {th }}$ centuries, the systems of differential equations needed to be able to describe all important physical phenomena, starting with the description and integration of individual movements. In particular, the laws of physics, first in the form of the Lagrange equations, later in the form of Hamilton's equations, must be capable of expressing the determination of any movement, any trajectory, hence of any physical event, in the same way as the laws of Newton-Laplace determine the evolution of celestial bodies in a gravitational field. And it is this equational determination which enables the predictions which measure the validity of theoretical propositions, at the center of the relation between experience and theory: observations are made, theories are produced (e.g. writing of the equations linking the observed actions and forces), predictions are made regarding the evolution of a system using these equations and finally, the predictions are compared against new observations. Effective predictions are the very objective of mathematical formalization.

The mathematical creativity of the new formalisms of the $X V I I I^{t h}$ and $X I X^{t h}$ centuries made scientist believe in a possible understanding of the whole Universe by dependable and progressive increases in knowledge. The ambition of the equations was to cover the whole world, to make it intelligible and predictable.

Of course, Laplace is also a great figure as regards the theory of probabilities, and this is no coincidence. He knew that many evolutions are random, thus unpredictable, for instance in the case of throws of dice which are submitted to forces and frictions that are too numerous to be known. These systems must then be analyzed in probabilities, in a completely different way than the methods specific to the equational determinations of movement. Laplace also knew that a deterministic trajectory may depend on "almost imperceptible nuances", for example as would a marble at the peak of a mountain (a maximum of potential) which, being submitted to unobservable ("imperceptible") perturbations, can take either one particular direction or a completely different one. Laplace nevertheless considered that such situations, such "critical" initial points, are isolated, that they are rare events in what concerns the measurement of space, and that it must certainly be possible to process them using adequate mathematics in the system which is a paradigm of stability and of certitude in terms of its predictability: the solar system.

It must be possible to deduce all astronomical facts,

according to Laplace. Besides, Alexis Clairault had even computed the time of Halley's Comet's return, an extraordinary achievement for the mathematics of the second half of the XVIII ${ }^{\text {th }}$ century. Determination and predictability govern the Universe, from particles to stars, with inevitable fragments of randomness - we are not omniscient - that must be analyzed in probabilistic terms which are quite distinct from those of systems of equational description. When known, such equational de- 
scriptions should always constitute, thanks to appropriate computations, the primary instrument of scientific prediction and of positive knowledge.

Well, they don't. Poincaré (1854-1912) demonstrated that it suffices to consider three celestial bodies, the Sun and two planets, say, under gravitational interaction for the system of equations describing the movement to become unable to predict the system's evolution. In the approach of this paper, we may say that the system of equations is epistemically "incomplete" with respect to knowledge as prediction of the physical process. Where is the problem? Newton had already realized this: his law of gravitation is "universal", meaning that it applies to the interaction between any celestial bodies; it even applies to the interaction between planets themselves. Therefore, if one can deduce from his equations the Keplerian orbit of a planet around the Sun, two planets also exert attraction upon one another and reciprocally disturb each other's movements. With time, these small perturbations can cause important changes, "secular" changes as Laplace would say, also being aware of the problem. And Newton had proposed the only solution likely to guarantee the stability of the system "in saecula saeculorum": once in a while, skillful adjustments by God reestablish order. Laplace, on the other hand, wanted to avoid any metaphysical hypothesis; he believed that a thorough mathematical analysis should demonstrate the stability of the system and its full predictability. It is thus that astronomers and mathematicians applied themselves during decades to resolving the equations of planetary movements; but when considering three bodies or more, they would encounter insurmountable difficulties.

In 1890, Poincaré noticed an error in his own demonstration of the convergence of Linsted's series. This series should have provided an analytical solution to the system of gravitational equations for three bodies (the "Three-Body Problem"). And, with all of his genius, he deduced from his own error the intrinsic impossibility of resolving this system. He demonstrated that almost everywhere, one obtains increasingly small divisors in the coefficients of the series, preventing convergence.

In an even more audacious and certainly innovating way, he gave a physical sense to this mathematical difficulty, to its "negative result", as he called it: radical changes for the evolution of three bodies can depend on very small (non-measurable) variations in the initial conditions - we will later speak of "sensitivity to initial conditions". Poincaré reaches this physical sense via geometry: he proves that in the "phase space" (of which the points are not only given by their position but also by the value of the momentum) trajectories present "bifurcations" points, while stable and unstable periodical trajectories intersect in an extremely complex way (in points that he calls "homoclines"). They indeed intersect infinitely often, in "infinitely tight" meshes and are also folded upon themselves "without ever intersect- 
ing themselves"1. Poincaré presents here deterministic chaos for the first time. He deduced then, as early as 1892, and later in a more developed way, that

prediction becomes impossible [...] and we have random phenomena (Poincaré, 1902).

It is often hard to give physical meaning to mathematical solutions of systems of equations; by first seeing it geometrically, Poincaré gave physical meaning to the absence of integral solutions.

To conclude, equational determination, here being relatively simple - only three bodies - , does not imply the predictability of the system. More precisely, the geometry of its evolutions enables to demonstrate this unpredictability as a consequence of its complexity. The homocline points, the bifurcations ... produce sensitivity to the system's initial conditions: fluctuations (internal) and/or perturbations (external) below observability can cause the system to have very different trajectories over time.

This work of Poincaré, which leads him to invalidate a program of knowledge, marks the beginning of the "geometry of dynamical systems" and of the qualitative analysis of unpredictable deterministic systems. It is mostly a topological analysis of global fluxes, of evolutions and limits, including quantitative ones, and of the limits of predictability (Charpentier et al., 2006). This will lead to the computation of the time necessary for a system, the solar system in particular, to become unpredictable, to be discussed next.

From an epistemological standpoint, the innovation of Poincaré's approach is to understand that random evolutions can even be found in systems of which the determination is relatively simple and that classical randomness can be understood as an unpredictable determination. A kay fact, largely ignored by common sense: determinism, or the possibility to fully "determine", by equations typically, may co-exist, it is not the opposite of randomness. A dice, a double pendulum or even a planetary system ... all of these are deterministic but chaotic systems as will be later asserted (see (Laskar, 1989, 1994) for the solar system). To describe a throw of dice, it would require numerous equations and it is not even worth it to attempt to write them: high sensitivity to initial or border conditions makes its movement unpredictable. But in no way does this change the fact that a thrown dice will follow a trajectory that is perfectly determined by the least action principle, a physical geodesic (a trajectory which minimizes the variation of energy over time), although it is unpredictable. Only two equations determine the movement of the double pendulum, but its evo-

${ }^{1}$ Poincare "sees" the geometry and the complexity of chaos, without even drawing it:

To represent the figure formed by these two curves [the stable and unstable periodic "trajectories"] and their infinitely numerous intersections [homocline points], these intersections form a sort of lattice, of fabric, a sort of network made up of infinitely tight meshes; each of these curves must never intersect itself, but must fold upon itself in a very complex manner so as to intersect an infinite number of times with all other meshes in the network. One is struck by the complexity of this figure, which I will not even attempt to draw. Nothing is more apt for giving an idea of the complexity of the three-body problem and in general of all problems of dynamics where there is no uniform integral (Poincaré, 1892). 
lution is quick to become chaotic and, therefore, is also unpredictable ${ }^{2}$. In what concerns the solar system, its time of unpredictability has been recently computed (Laskar, 1989, 1994). If we associate, to the detailed analysis of (non-linear) movement equations, a lower bound for the best measurement possible, we obtain, by non-obvious computations, an upper bound for predictability. This bound is relatively modest in astronomical terms (a few tens of millions of years, depending on the planet). So, for modern dynamics, from Poincaré onwards, the solar system is chaotic. Let's note however that there are those who, having understood this a little late, even felt compelled to apologize on behalf of a whole scientific community. This was done, in a very British way, in a famous and mathematically very interesting article, but without referring to the previous century's illustrious French mathematician otherwise than as the source of an error (Lighthill, 1986) ${ }^{3}$.

As a consequence, we insist, classical randomness, seen as the unpredictability of a physical process, is a specific case of determination: that which governs a chaotic deterministic system. One could bet on the odds that Earth will still be in orbit around the Sun 100 million years from now: it is roughly as unpredictable as a throw of dice relative to its own time scale. One will note that "chaoticity" is a precise mathematical property, defined in general by means of three well formalized properties that have been clarified with rigor and with full generality after 1970 (sensitivity to the initial conditions, existence of dense orbits, density of periodic points $)^{4}$.

\footnotetext{
${ }^{2}$ A pendulum can be conceived as a bar connected to a pivot. If we attach another bar to the bottom of this first bar, one that is also free to rotate, what we have is a double pendulum. A recent and amusing theorem (Beguin, 2006) demonstrated the following: if we choose a sequence of integer numbers $a_{1}, a_{2}, a_{3}, \ldots$, we can put the double pendulum in an initial position so that the second limb will make at least $a_{1}$ clockwise turns, and then change direction to make at least $a_{2}$ counterclockwise turns, and then at least $a_{3}$ clockwise turns, etc. If we choose a random sequence $a_{1}$, $a_{2}, a_{3}, \ldots$ (see section 6), this purely mathematical result makes chaos and unpredictability "understandable" (but does not demonstrate it) in one of the simplest deterministic systems possible. We can also see this by observing an actual physical double pendulum or a computer simulation (such simulations can be found on the Web, we will return to this).

${ }^{3}$ It is interesting to compare explicit theorization and geometric analysis of the unpredictability of a Newtonian system in (Poincaré, 1892) and the references (as well as the title! "recently"?) in (Lighthill, 1986). Indeed, two schools particularly distinguished themselves in the $X X^{\text {th }}$ century regarding the theory of dynamic systems: the French school (Hadamard, Leray, Lévy, Ruelle, Yoccoz ... ) and the Russian school (Lyapunov, Pontryaguin, Landau, Kolmogorov, Arnold ... ). To these, we must add, namely, the Americans Birkhoff and Lorentz. But works on the subject by Hadamard, Lyapounov and Birkhoff have long remained isolated and seldom quoted. Up until the results by Kolmogorov and Lorentz in the 1950s and 1960s, and well beyond, Classical Rational Mechanics - of which Lighthill presided the international association in 1986 - has dominated the mathematical analysis of physical dynamics (as well as the way the subject matter was taught to the author of this article, prior to such apologies, alas).

${ }^{4}$ (Ruelle et al., 1971) faced many difficulties for getting published. As has been said above and as will be said again as for genocentric approaches in Biology, the Laplacian mentality (but Laplace, two centuries ago, was a great mathematician) is still present in many minds of today, although outside of the sphere of Mathematical Physics. And, in general, "negative results" are the most difficult to accept. Thus, they are the most difficult ones to finance, even if they are most likely to open up new horizons. And this is exactly what the institutional administrators of research steered
} 
In most cases, from a mathematical point of view, chaos appears when the system's evolution equations or evolution function are non-linear. It is the typical mathematical means of expressing interactions and effects of attraction/repulsion or of resonance (technically, two planets enter gravitational "resonance" when they are aligned with the Sun; it is a situation where there are great mutual perturbations). Unpredictability is, for its part, a problem at the interface between the mathematical system and the physical process, via measurement: if the mathematical description of a physical process (the equations or a function which describes its evolution) verifies the formal conditions of chaos, it is the physical process which becomes unpredictable by this mathematical system. A measurement, in Classical (and of course Relativistic) Physics, is indeed always an interval, it is always an approximation. Because of this, non-measurable fluctuations or perturbations (within the best measurement's interval of approximation) can entail, over time, changes which are quite observable, but which are unpredictable. In other words, in order to predict or to demonstrate that it is impossible to predict, it is necessary to view a physical process mathematically. If the determination produced by the mathematical approach is "sensitive to initial or border conditions" (a crucial mathematical property of chaotic systems) and if the measurement is approximate, as always in Physics, then unpredictability appears.

We will later indicate how it is possible to relate, from both an epistemological and technical point of view, the unpredictability of deterministic systems to the Gödelian undecidability of logico-formal systems. From a historical standpoint, it is easy to see a first analogy (we will see others). The new conception of the physico-mathematical "determination" which stems from Poincaré's negative result, this limit to equational knowledge, as well as its qualitative geometrical analysis, have paved the way for the geometry of modern dynamic systems. Analogously, Gödel's theorem, setting a limit to formal knowledge, marks the beginning of contemporary Mathematical Logic (Computability Theory, Model Theory and Proof Theory). The epistemological fracture - as Bachelard puts it —, of great importance and very surprising at the time (and often still so today), caused by each of these great negative results, was extraordinarily fruitful in science ${ }^{5}$.

towards positive projects and towards patents will succeed in hindering even more definitively, bolstered by their bibliometric indices: the role of critical thinking and of intrinsically "anti-orthodox" innovation that are characteristic of scientific research, see (MSCS Ed. Board, 2009) and (Longo, 2014), (Longo, 2018d).

${ }^{5}$ Besides those already mentioned, there are numerous other highly important "negative results", particularly in Physics (not to mention that in Mathematics, by a skillful use of double negations, any result can be presented as "negative"). The results of which it is question here are among those which contradicted major projects of knowledge, or theories that marked the history of science and which sometimes continue to guide common sense. They are also results that are linked to the negation of an assumed completeness (in its various forms) of these theoretical propositions. 


\section{From Geometry to Logic}

The program for the progressive and full occupation of reality using formal writing has an epistemological parallel in the formalistic "creationist" view and an illustrious predecessor in the person of George Berkeley. The English bishop was particularly impressed by the invention of complex numbers, and by this imaginary " $i$ ". Such an audacious linguistic and symbolic notation, enabling to resolve an equation without "real" solutions, led him to conceive of the mathematician as a creator of formal instruments for comprehension serving to gradually construct knowledge. According to Peacock (1830) and Peano (1889), first came $\sqrt{2}$, which goes beyond the ratios between integers, introduced for the purpose of understanding the diagonal of a square, and then came $\pi$ for understanding the circle: these formal inventions allowed new mathematics and new understanding or computations. And so the algebraically complete field of complex numbers was progressively reached, gloriously culminating by the invention of the imaginary " $i$ ": any algebraic equation has a solution in it.

Hilbert made use of these considerations in the context of a deep analysis of the foundations of Mathematics. He sought formal systems which are demonstrably consistent and complete, which he will designate in the 1920s as constituting a "definitive solution" to the foundational problem that was such a humongous issue. Thankfully, in science, there is no such thing as a definitive/final solution.

But which foundational problem? It is certainly not a question of these antinomies of the beginning of the century concerning a barber who shaves all those who do not shave themselves (must the barber shave himself?), Sunday amusements and contradictions at the barber's shop that are (and were) easily resolved. The mathematical practice (or "doxa") is "typed": in mathematics, we do not generally authorize the barber to shave himself, no more than we allow functions to apply to themselves. We start by defining functions over natural and real numbers, having values within these (or other) "types" of numbers; then we define functionals over functions, for example, the integral, and we continue in a hierarchical manner. A formalization which frees itself from meaningful precautions, failing to take them into account, easily leads to contradictions. This happened several times: such attempts are a part of research ${ }^{6}$. These antinomies (formal contradictions) do not deserve however the designation of "paradox" (against the "doxa", seen as common "mathematical" knowledge), a designation rich in history since Ancient Greece, and even less so when the doxa already contains the solution. One only needs to think

\footnotetext{
${ }^{6}$ We can, for example, recall the first formalization of one of these fundamental systems of computability, Church's untyped lambda-calculus (1932). The ensuing "Curry paradox", an antinomy similar to that of the barber's, will first entail a refinement of the calculus (1936) and the invention of another one, with "types" (1940). The first formal system of types by Martin-Löf will also be contradictory (1970). The formalizations, which loose "meaning" along the way (we will return to this), easily tend to produce contradictions: "Logic is not sterile", said Poincaré in 1906, "it has created contradictions". Given the innovations they brought and the responses they were quick to receive, it must be noted that these formal theories, in spite of these errors of syntax due to the lack of a mathematical interpretation, were at the origin of very interesting ideas and systems and not of a major crisis, except among logicists (Longo, 1996).
} 
about Zeno's paradox, which constitutes a true challenge to the doxa and which opened century-long discussions.

The true problem of the foundation of mathematics was rather to be found in the collapse of the Euclidean intuition of space, the Newtonian absolute in Cartesian coordinates. For over two thousand years, Euclid's "Elements" had provided the link between the geometric constructions in sensible space and physical space on any scale. The theorems which were constructed in such space with the intuition of our world of the senses and of action produced both the geometric relationships between the stars and between Democritus's atoms. For Kepler, Newton and Kant, sensible intuition like mathematical intuition was at the roots of this geometric reconstruction of the universe. And this universe was described, as Galileo had said, using the language of Euclidean circles, triangles and straight lines.

Well, such is not the case, proved the geometers of the $X I X^{\text {th }}$ century: the interesting space manifolds are not "closed under homotheties". What does this mean? Riemann, in his habilitation of 1854, proposed a general framework for what we call non-Euclidean geometries. In short, by following the algebraic treatment by Klein (1872), we can observe that one of their crucial properties is the fact that the group of automorphisms (internal transformations or symmetries) does not contain homotheties - that is, arbitrary changes of size. In Riemann's geometry, it is possible that the theorem regarding the sum of a triangle's internal angles - which is equivalent to Euclid's axiom of parallels - gives more than $180^{\circ}$ when the triangle is expanded to a stellar order of magnitude. Moreover, Riemann conjectured that the "forces of cohesion between bodies are linked to the metric of space" when he demonstrated the general theorem of the metric tensor which in turn links the metric to the curvature of space. Einstein will give specific physical meaning to this audacious negation of the Euclidean universe, by the role of the distribution of energy and matter in structuring (the curvature of) relativistic spaces. Locally, in "tangent" planes of null curvature, Euclidean geometry provides a good approximation; but on a global scale, on the scale of the Universe, it is precisely the non-null curvature which enables to unify gravitation and inertia, the keystone of Einstein's relativity.

The non-null curvature of space, its metric structure ... a revolutionary geometrization of physics, originated from the "negation" of the Euclidean doxa. "A delirium", Frege will say in 1884, which made Riemann and his followers to renounce to the Cartesian intuition in Euclidean spaces. As for a conceptual and intuitive analysis, those spaces were the only possible ones for Frege, to a point where he will continue even after 1920 to think of the foundations of geometry in Euclidean terms. But prior to that, at the very end of the $X I X^{\text {th }}$ century, in reaction to the non-Euclidean frenzy which marked the genuine crisis of all certitudes in mathematical intuition, he established the basis of a new mathematical discipline, one that is important and rigorous: modern Mathematical Logic.

Of course, numerous other people participated in this work, among whom Peano. But Frege is the first for whom the foundational preoccupation radically emancipated itself from the relationship to sensible and intuitive space, in order to focus on logico-deductive analysis, constructed using the founding (and absolute) concept of integer number and a rigorous treatement of "quantification" (for all ... there exists 
... ). Arithmetics is logical; the principle of induction (or of recurrence), formalized by Dedekind and Peano, is a logical principle which fully captures, and even identifies itself to the conceptual structure of integers.

The extraordinary developments of this foundational approach are before our eyes: logical arithmetic machines change the world. They are the direct products of a mathematical and philosophical work initiated by English algebraists such as Peacock and Boole, and reaching Frege, Peano, Hilbert, Gödel and finally, Turing, all focusing on (formal) Arithmetics and Logic.

\section{From Hilbert to Gödel}

Hilbert as well was foremost preoccupied by the loss of certitude due to the nonEuclidean shift in Geometry. His most important foundational text, the Foundations of Geometry (1899), sets the basis for an original approach to the question, well beyond the algebraic unification of geometries proposed by Klein. Perfectly abstract axiomatics must formally capture the various systems, while ensuring the mathematical transformations of each of them and while revealing the "foundational" properties from which to derive all theorems of each system. It is a calculus of signs, freed from the incertitude of the intuition of space, based on axioms and rules of deduction of which we could "potentially" mechanize the application. It is the locus of mathematical certitude, precisely because it is devoid of meaning, of spatial signification, which is a source of ambiguity and the locus of space intuition, which had turned out to be rather unreliable.

During the following years, "formalists" will insist that certitude resides in the formal manipulation of finite sequences of signs, on account of rules that are also described by finite sequences of signs without any semantic and intuitive reference. Given a "sequence-of-signs rule", such as "from $A$ and $A \rightarrow B$ follows $B$ ", formally deduce $B$ from $A$. That is, by applying the rule, if the first $A$ is (constituted by) a sequence of signs identical to those in the second $A$, write/deduce $B$. What is the meaning of $\rightarrow$, the arrow? It doesn't matter: a machine must be able to apply this formal schema of deduction.

So, the existence of mathematical objects is not, for Hilbert, an ontological question: it is ensured by the sole consistency of the axiomatic system within which they are defined, that is, by the impossibility of deducing a contradiction from the axioms by using the system's rules of deduction. In other words, if (it is possible to demonstrate that) a system is non-contradictory (consistent), then hypotheses and existence proofs, even proofs by contradiction, are the guarantee of existence specific to the objects of Mathematics. It is a strong and bold choice, a veritable turning point by its rigor and its clarity of exposition with respect to the ancient and ontological myths of ideal triangles and circles that "exist" because they are present in the mind of God. For Frege, instead and in spite of his error in formalizing Set Theory, mathematical signs and properties must make sense, must evoke in language meaningful or derivable concepts. And it is he who will oppose, in a polemic manner, 
an axiomatic theory of the properties of God that is non-contradictory; he ill then will observe that he has thus proven God's existence. This is not what Hilbert had in mind, infuriated by Frege's observation. He was talking about a formal deductive practice, one that is purely linguistic and specific to Mathematics and to its objects, with no ontological content in Frege's sense.

So, how then may the consistency (the non-contradiction) of axiomatic theories be demonstrated? Hilbert, in his book, translates or "interprets" the various geometric axiomatics, including Riemannian axiomatics, within the continuum of Analysis, which can be constructed from Arithmetics, following Cantor-Dedekind's method, yet another fantastic achievement of late $X I X^{\text {th }}$ century. He thus observes that if it is possible to prove the consistency of Arithmetics, the analytic interpretation guarantees the consistency of all axiomatized geometries. This is why he posed, in a very famous conference presented in Paris the following year (1900), the question of the consistency of Arithmetics (and of Analysis) as being among the great problems for $X X^{\text {th }}$ century Mathematics. And he was right, given the consequences that this problem will have and the importance of the formal mathematical framework proposed. His work on the formalization of geometries, highly rigorous, marks indeed the birth of the Axiomatic Method, one of the most fruitful methodological turning points of the $X X^{\text {th }}$ century.

\section{1 ... Through Poincaré and Weyl}

Poincaré reacted strongly to Hilbert's bias and wrote a lengthy review of Hilbert's 1899 book. He indeed appreciated its technical novelty and profoundness, but not the foundational vision of Mathematics as a mechanical issue and practice devoid of reference to signification.

It is viewed, he noted, as a "mechanical logic piano" which produces theorems in a purely formal way:

[...] a machine might be imagined where the assumptions were put in at one end, while the theorems came out at the other, like the legendary Chicago machine where the pigs go in

alive and come out, transformed into hams and sausages

he would write in (Poincaré, 1908). And the rift between the two visions will widen over time. Hilbert, as we said earlier, will develop — in his own way, given his scientific originality - this formal linguistic tradition which relied on the set of signs, be they new or devoid of meaning (like the imaginary " $i$ "), to practice and broaden Mathematics. On the one hand, the potentially mechanical manipulations of signs should be the locus of the certitude of Mathematics. On the other hand, as we will clarify later on, the completeness of sound formalisms will guarantee the possibility of reducing all Mathematics to the formal method. And we see once more the trace of the old positivist program. The formal systems of Laplace equations should cover the world, should fully explain its determination and predict its evolution ("by allowing to deduce all astronomical facts", said-he). Thus, any question regarding future astronomical evolution, in deterministic systems such as the 
solar system, must have an answer. In the same way, any mathematical problem should, for Hilbert, have a solution, an answer: yes or no. In particular, the formal system of Arithmetics should be complete: each of its assertions, as long as it is well-formulated, should be decidable.

Of course, among possible answers, we could have impossibility results. The Greeks were able to provide such results regarding the impossibility of expressing $\sqrt{2}$ as a ratio of integers; the transcendence of $\pi$ (the impossibility of an algebraic representation) had been recently demonstrated. But Hilbert's conceptual reference, the theoretical example towards which he aspired, was the same as for his predecessors, particularly Peano: it was the complete field of complex numbers. The audacious formal maneuver, the invention of an " $i$ ", was devoid of meaning and represents, for this school of thought (the formalist school), the paradigm of the practice and creativity of Mathematics. If we extend the field of real numbers using this meaningless sign, then, within the field of complex numbers it generates, we obtain algebraic closure or "completeness": any algebraic equation will admit a solution, as we said. "Non ignorabimus" in Mathematics, as Hilbert stressed at the Paris conference of 1900. At most, it will be a question of extending the chosen formal system, in a consistent way, with new notions and principles of proof that are well formed: this will allow to answer any purely mathematical question.

Poincaré will disagree with Hilbert's approach in several texts: unsolvable problems, those which are demonstrated to be unsolvable, exist and are the most interesting because they open up new avenues. We can add that there does not exist any formal extension of the Newton-Laplace equations which enables to predict the evolution of three bodies. Of course, Poincaré could not use this argument against Hilbert, because deterministic unpredictability is born at the interface between an equations' system and a physical process which the system describes, via measurement. This is not a "purely mathematical" problem, as are the existence of solutions, the equality between two formally defined functions or an assertion of Arithmetics ... Hilbert believes that these problems must always find a solution, even if it is possible to obtain impossibility theorems, such as the irrationality of $\sqrt{2}$. The latter are only stages for attaining a complete theory which would answer any well-formalized mathematical question. Of course, such a theory must be consistent: in a contradictory system, anything and its opposite can be demonstrated. And since certitude resides in finitude, it resides for Hilbert only in the formal play of signs, in a combinatorial calculus on finite sequences which we could mechanize, it is also necessary for the proof of consistency to be finite. In other terms, a rigorous demonstration is composed of finite deductions, of finite sequences of signs, of formal statements, line by line. By their mechanical character, they remain far removed from the ambiguities of meaning. So for the first theory of Mathematics, Arithmetics or Formal Number Theory, to which Hilbert had reduced the various geometric axiomatics, a proof of consistency must also be obtained by means of a finite formalism, that is of a finite analysis of sequences of signs, line by line. It would thus have been possible to ensure at once the non-contradictory character of Number Theory and of Euclidean and non-Euclidean geometries. 
During a 1904 symposium, Hilbert proposed a program for the proof of this consistency, a schema based on an inductive analysis of the formal proofs of Arithmetics. Not without irony, Poincaré observed in 1906 that "Monsieur Hilbert" thought to demonstrate by induction the consistency of Arithmetics, of which the main axiom is induction! For more than ten years, Hilbert will be less interested in the problem of foundations, to the greatest benefit of Mathematical Physics and Analysis to which he will contribute very significantly. Besides, the "best among his students", the great geometer, physicist and mathematician Hermann Weyl, will also during these years distance himself from Hilbert's foundational philosophy. In his book The Continuиm (1917), Weyl explains several times that Mathematics is rendered trivial by the idea of its potential mechanization and of its decidability, by all demonstrations made "with fixed deductive techniques and in a finite number of steps". And above all, in a way that is uncertain, confused, and arguably hesitant (how would one dare thinking in opposite of his great professor?) he conjectures the incompleteness of Arithmetics (1917, end of Sect. 4). He will later on define himself as a "lone wolf".

Hilbert's steadiness regarding his program is exemplary indeed. In the beginning of the 1920s, he returns to his proof by induction of the consistency of Arithmetics using another framework: "meta-mathematical" induction. Throughout all of these years, he stresses an important distinction: a formal system is a very specific fragment of mathematics whereas the mathematical work one can do upon it is metamathematical. In other words, Meta-Mathematics has for object of study the formal axiomatic systems, particularly insofar as they can be examined as finite sequences of signs. One must note once more the originality of Hilbert's vision: from 1900 to 1922 (and even in 1928, during a famous Mathematics symposium in Bologna), he proposed a meta-mathematical analysis of mathematical deduction, described as an algebraic-combinatorial calculus. This approach to the question of the foundations of Mathematics is truly innovating. In what concerns his proof of consistency, however, it is Weyl (Poincaré being deceased) who will point out to him that his proof by meta-mathematical induction nevertheless remains a proof by arithmetic induction. It can therefore not serve as "foundation" for a theory of which the axiomatic core is induction. Wittgenstein will insist in 1929:

Hilbert's Metamathematics must necessarily turn out to be Mathematics in disguise.

And because a meta-mathematical proof should be

[...] based on entirely different principles w.r.t. those of the proof of a proposition [...] in no essential way there may exist a meta-mathematics.

Therefore:

I may play chess according to certain rules. But I may also invent a game where I play with the rules themselves. The pieces of the game are then the rules of chess and the rules of the game are, say, the rules of logic. In this case, I have yet another game, not a metagame. (Wittgenstein, 1968, $§ 153$ and p. 315).

As we will see, Gödel will shatter, both mathematically and within Formal Number Theory, the foundational role of this distinction between theory and meta-theory, by 
encoding the latter as part of the former. This distinction is useful from a technical standpoint, but it is artificial, or at least it tends to exclude from the Hilbertian framework the epistemological aspects of the foundations of Mathematics; we will return to this.

\subsection{Arithmetics, an Absolute}

Arithmetics, as a (formal) Theory of Numbers, is very important in Mathematics and occupies, no less according to Hilbert than to Frege, a central place in the search for foundations. However, the gnoseological frameworks used by these two founding fathers are completely different. For Frege, ultimate certitude resides in the signification of natural numbers understood as concepts, as logical and ontological absolutes. For Hilbert, conversely, it resides in Arithmetics as the locus of the finite, which can be counted or written using a finite set of signs and which has finitude as its very object of study. Both start with the problem of space, of the crumbling of Euclidean certitudes. But Hilbert, being one of the great mathematicians and geometers of his time, wishes to save non-Euclidean geometries. It is his main objective, as shown by his 1899 book, contrarily to Frege. Hilbert, by the relative consistency proof passing by Arithmetic, refers to finitistic/mechanical formalisms as a tool for solving once and for all the problem of foundations and, then, at last, working freely and safely in "Cantor's paradise of infinities". Both authors nevertheless propose a new absolute reference for foundational analyses: Arithmetics.

Indeed, the consistency of Mathematics itself would have been guaranteed if Hilbert's program had succeeded. In order to be certain, a proof of consistency of Arithmetics had to be itself formal, finite and therefore arithmetizable. Now, Arithmetics - the Theory of Integer Numbers enables the encoding of everything which is finite, as Gödel will formally demonstrate. Arithmetics would then have removed itself from the intuition of counting and ordering in time and in space, thanks to finite (arithmetic) computations using pure formal signs; it would have elevated itself over the world by itself, by pulling "on itself", just as the Baron of Münchausen would lift himself up by pulling on his own hair. It would have become the place of ultimate certitude, without recourse to meaning. This perfectly formal and closed locus of deductive certitude, capable of self-demonstrating its own consistency, would have been an absolute that was both different and parallel to the ontological absolute of Frege's concepts and numbers. Hilbert actually speaks of an absolute formal proof of the consistency of Mathematics as part of the definitive solution to the problem of foundations. For those who consider Mathematics to be central from an epistemological and cognitive stand-point, this program proposes the definitive foundation of all knowledge,

to make a protocol of the rules according to which our thinking actually proceeds,

Hilbert asserts in the The Foundations of Mathematics published in 1927. 


\section{The Theorem}

Well no, it doesn't work, even not for thinking (proving) properties of integer numbers. If Arithmetics (Formal Number Theory) is consistent, i.e. it does not prove a contradiction, then not only is it incomplete - meaning that there exists in its language assertions which are undecidable, that is that can not be proved and of which the negation is also unprovable — but it is also impossible to complete: it has no consistent and complete formal extension - Arithmetics is "non-completable". The analogy with the algebraically complete field of complex numbers does not work: it is impossible to add signs or formal axioms to define a complete (or maximal) theory which contains Arithmetics. But there is more: the consistency of Arithmetics, when it is formalized, in the manner of Hilbert one might say, can not be demonstrated within Arithmetics. In other words, there is no finitary proof of consistency for Arithmetics. That is, in a few words, Gödel's results represented a true cold shower on formalist ambitions, which some will still try to save by introducing different variations and modulations to the notion of "finitary proof". We will indeed see how it is possible to "lengthen" finite induction along infinite ordinals in order to improve the situation and, in a technically interesting way, to set a hierarchy between theories and to shift the problem of consistency from theory to theory. It nevertheless remains that "non-completability" is provable and is intrinsic to the system. This signals the death of the possibility for an ultimate foundation of Mathematics on an absence of meaning, on a potentially automatable computation of signs. The "non-completability" result is a difficult and schocking fact, still to be digested by many.

Let's examine a few technical points of Gödel's proof, without going into the details of the proof of the first theorem, which is a formal masterpiece. But before this, one remark must be made. Gödel never used, neither in his statements nor in his proofs, the notion of "truth", which is not a formal concept. It is necessary to stress this point, because in current readings of this theorem, it is often too hastily said that it shows the existence of "statements that are true but unprovable" in Arithmetics. "True" statements? But where, how, according to which notion of truth? This is a delicate question to which we will return, avoiding Platonizing flights of fancy postulating a list of true statements that already exist in the mind of God, but among which some are "unprovable". Such ramblings have nothing to do with Gödel's proof. The strength of his work is, to the contrary, of shattering the formalist program from the inside, using formal tools. He uses pure computations of signs without meaning and therefore does not invoke "transcendental truths"; he presents his argument by purely formal means. We can see a first analogy with Poincaré's Three Body Theorem, a result which demolished the myth of an equational determination capable of fully predicting the evolution of the world, and this was also done from "within", by means of a purely mathematical analysis of the equations, that is of their non-integrability, only later followed by an original geometric and physical interpretation of this. Of course, also Gödel's theorem needs to be (correctly) interpreted. 
The first among Gödel's great ideas was to encode, using numbers, all propositions of any formal system given by a finite number of finite sequences of signs, in the form of axioms and of rules of deduction. In particular, by numbering each sign and each letter of the language of Arithmetics, Gödel bijectively associated a natural number-code to each statement of Arithmetics as formalized by Dedekind, Peano, Frege and Russell (which we will call PA, for Peano's Arithmetics).

We do not need to go into the details of this formalization which rigorously describes the well-known axioms ${ }^{7}$, and even less so into the details of its encoding (which we call "Gödelization" or Gödel Numbering, see (Kreisel, 1984) for a discussion on a category-theoretic understanding of this fundamental notion and more references). Today, these numerical encodings of letters are, indeed, everywhere. By the Gödelization of propositions, of sentences, but also of music and images, logico-arithmetic machines enrich and transform our existence. All the sentences that you read from your computer screen are encoded using binary integers, just as Gödel proposed to do for the assertions of any formal language. We will then designate here as $\underline{A}$ the Gödel number of the proposition $A$. For example $2=1+1$ is a proposition, whereas $2=1+1$ is its Gödel number, let's say 651847 , or the number which digitally encodes in this author's computer memory this proposition as displayed on its screen. Gödel will thus be able to mathematically address the until then informal notion of "effective" or potentially automatable deduction. The deduction of formulas from other formulas, such as of $2=1+1$ from the axioms of $P A$, written as " $P A \vdash 2=1+1$ ", will be treated as a function associating numbers to numbers (the Gödel Numbers of such formulas). It is therefore a calculus of formal signs. To do this, he describes a class of functions defined by the computations one can finitely and effectively describe in $P A$ if one considers, as did Hilbert, that $P A$, formalized Arithmetics, is the locus of finitist effectivity. These functions use for basis the constant function 0 , the successor function "Succ", and almost nothing else. From here, one defines by induction the operations of sum and product, as well as a huge class of arithmetic functions, the computable or (primitive) "recursive" functions. There already existed definitions of such functions, but Gödel completed and stabilized their definition with great rigor.

So, we write " $P A \vdash B$ " to say that proposition $B$ is deduced from the axioms of $P A$, that is, that $B$ is a theorem of $P A$. Gödel then constructs, by induction over the structure of formulas, functions and predicates in $P A$ which encode the formation and deduction of formulas from $P A$. For example, he defines the primitive recursive functions neg $(x)$ or $\operatorname{imp}(x, y)$, which represent in $P A$ the negation of a formula or the implication between two formulas through the Gödelization of these formulas. In other terms, neg $(x)$ and $\operatorname{imp}(x, y)$ are functions, written in the language of $P A$, such that :

$$
P A \vdash \operatorname{neg}(\underline{A})=\underline{\neg A} \quad \text { and } \quad P A \vdash \operatorname{imp}(\underline{A}, \underline{B})=\underline{A \rightarrow B} .
$$

\footnotetext{
7 The properties of 0 and of the successor symbol ( 0 is not a successor and the successor operation is bijective) and, especially, of induction: suppose $A(0)$ and that from $A(n)$ one is able to deduce $A(n+1)$, then deduce $A$ for all integers, i.e. $A(m)$ for all $m$.
} 
Thus, Gödel encodes the operations of construction and of deduction of the formulas of $P A$ until reaching a predicate of $P A$, which is written $\operatorname{Prov}(x, y)$, such as $\operatorname{Prov}(\underline{A}, n)$ represents or encodes the fact that the formula $A$ is provable, from the axioms of $P A$, using the finite sequence of formulas represented by their Gödel number $n$.

The reader will notice the gradual emergence of a huge tide of circularity. Indeed, we have just quickly seen how to define in $P A$ deductions over $P A$. So we see how to write a predicate $\operatorname{Theor}(\underline{A})=\exists y \operatorname{Prov}(\underline{A}, y)$ which encodes the fact that "there exists" as $\exists$, in $P A$, a proof of $A$, that is the (Gödel) number of a proof " $y$ " of $A$, or that $A$ is a theorem of $P A$. This predicate is a predicate on numbers, because it is numbers that are the objects of $P A$.

More formally, Gödel's great feat in terms of encoding and of computation enables him to write an arithmetic predicate Theor and to demonstrate that:

$$
\begin{aligned}
& \text { If } P A \vdash B \text {, then } P A \vdash T h e o r(\underline{B}) \text {. } \\
& \text { If } P A \vdash \text { Theor }(\underline{B}) \text {, then } P A \vdash B \text {. }
\end{aligned}
$$

In other words, point (1) states that if $B$ is a theorem of $P A$, this very fact can be stated and proved within $P A$, in the sense that also Theor $(\underline{B})$ is a theorem - the metatheory, i.e. the provability of $B$, gets into the theory. Point $(2)^{8}$ says the opposite: if one can prove within $P A$ that $B$ is a theorem, i.e. if Theor $(\underline{B})$ is proved, then $B$ is indeed a theorem of $P A$ - the coding of the meta-theory in the theory is sound. Another formulation: Theor $(\underline{B})$ is nothing else than the writing in $P A$ of the function that computes the "effective deduction" of $B$ from the formulae-axioms of $P A$.

Another step, and we will have closed the loop of circularity. We write as $\neg B$ (not- $B$ ) for the negation of $B$ in $P A$. Then, all we need to write, thanks to non-obvious ingenuities of computation, deduction and recursion, i.e. fix points, is a formula $G$ such as:

$$
P A \vdash(G \leftrightarrow \neg \text { Theor }(\underline{G})) .
$$

Let's now suppose that $P A$ is consistent, i.e. that it does not generate any contradiction (it is impossible, for any $A$, to prove both $A$ and $\neg A$ ). We then demonstrate that $G$ is not provable in $P A$. If it was, that is, if $P A \vdash G$, point (3) would imply that $\neg$ Theor $(\underline{G})$ is provable (that is, $P A \vdash \neg$ Theor $(\underline{G})$ ). Now point (1) states that, from $P A \vdash G$, one can also derive $P A \vdash$ Theor $(\underline{G})$. Contradiction.

But we can show that $\neg G$ as well is not provable. One just needs to use the rule of "contraposition" that is formalized, for any theory $T$, by

$$
\text { (Contrap) : } \quad T \vdash(A \rightarrow B) \text { implies } \quad T \vdash(\neg B \rightarrow \neg A),
$$

and the result of classical logic $P A \vdash(\neg \neg A \rightarrow A)$. Point (3) can then be rewritten as $P A \vdash(\neg G \leftrightarrow T h e o r(\underline{G}))$. So a proof of $\neg G$ gives a proof of Theor $(\underline{G})$, and therefore a proof of $G$ by point (2). Contradiction.

\footnotetext{
${ }^{8}$ Point (2) requires a hypothesis only slightly stronger than consistency: $\omega$-consistency. This is a technical, yet very reasonable - and natural — hypothesis: the natural numbers are a model of $P A$ — it would be "unnatural" to assume less.
} 
In conclusion, we constructed a proposition of $P A$ which is undecidable: not provable itself, and its negation not being provable either. So if $P A$ is consistent, it is incomplete.

Formula $G$ is a sequence of signs, which we produced rigorously using a pure computation on signs; that should suffice for us. But it is possible to give it an intuitive "meaning". The reader, whose brain is not a Turing Machine, will thus be able to informally "understand", attribute a suggestive "meaning" by a formally inessential abuse, to this proof, whose formal construction we rapidly presented. By (3), formula $G$ "says" that " $G$ is not provable". That is, $P A$ proves that $G$ and its unprovability, formalized by $\neg$ Theor $(\underline{G})$, are equivalent. We are thus forcing meaning where there is none - and where, formally, there there is no need to be any: (one proves that) $G$ is (equivalent to) the sentence " $G$ is not provable".

The analogy with the liar paradox ("this sentence is false") is obvious: just replace "false" by "unprovable". In his paper's introduction, Gödel also acknowledges this brilliant invention of Greek culture as one of his sources of inspiration. But to obtain this contradiction, one must not refer to meaning (true/false) as in the liar paradox. To the contrary, it is necessary to remain within the formal theory of proofs and challenge the (provable/unprovable) contrapposition. This is what Gödel does with great rigor. Now "this sentence is false" is neither true nor false, and there lies its great force and its paradoxical nature. Likewise, $G$ will not be provable, nor will its negation be, if we suppose that $P A$ is consistent.

But what have we used from $P A$ ? Only its capacity to encode propositions and formal proofs. So any sufficiently expressive formal theory $T$, that is, one that can be axiomatized (thus encoded), and which contains $P A$, enables to construct an assertion $G T$ that is independent from $T$, if $T$ is consistent. Thus, for any consistent extension $T$ of $P A$, undecidable propositions exist for $T$. $P A$ is therefore impossible to complete: there is no "field" (consistent formal theory) which is complete (maximal) and which contains Arithmetics, to use once more the inspiring analogy with the algebraically closed (complete) field of complex numbers. As regards mathematical theories which do not contain $P A$, they do not know how to count using integers: generally, we can't do much with them (induction pops out everywhere in real mathematics).

During this brief overview of encodings and contradictions, we had to omit details that are essential (and that are sometimes, but not always, mathematically difficult). The very technical aspect of the First Theorem, encodings and formal deductions, span several pages, does not afford us, in a text such as this one, the possibility of delving further into it. But we are not however done with our ponderings: there is a Second Theorem of incompleteness. What does it mean in formal terms that $P A$ is consistent?

As we have seen, a (mathematical) theory is consistent if it does not generate contradictions: it is impossible for any $A$ to prove both $A$ and $\neg A$. Indeed, only one contradiction is enough to deduce everything from it: "ex falso quodlibet", as my Roman ancestors used to say. $P A$ is therefore already contradictory if we only demonstrate $0=1$, which negates one of the axioms. It is then easy to state in $P A$ that $P A$ is consistent: all one needs to do is to write $\neg$ Theor $(\underline{0=1})$. So the 
proposition Cons $\equiv \neg$ Theor $(\underline{0=1})$ is a formula of $P A$ which states the consistency of $P A$, by saying formally that $P A$ does not demonstrate $0=1$. One must note the strength of the formalism: Cons soundly describes in the theory $P A$ the eminently meta-theoretical (meta-mathematical) assertion " $P A$ is consistent", or $P A$ does not entail a contradiction. If we prove that $C o n s$ is not provable in $P A$, then we will have proven the impossibility of demonstrating the consistency of $P A$ using methods that are formal and finite, and therefore encodable in $P A^{9}$.

Building upon all the techniques produced while addressing the first theorem, Gödel proved the second in a few lines. These lines are however extremely dense and abstract. There is no longer any need (nor even any mathematical opportunity) for interpretation. All is based on the syntactic proximity between the $\neg$ Theor $(\underline{G})$ and $\neg$ Theor $(0=1)$ formulas. And as a result of a few very formal (and meaningless) lines, the second incompleteness theorem demonstrates:

$$
P A \vdash(\text { Cons } \leftrightarrow G) .
$$

In other words, in $P A, C o n s$ and $G$ are proved to be equivalent. Of course, the implication that interests us most here is: $P A \vdash($ Cons $\rightarrow G)$. That is, in $P A$, one may formally deduce $G$ from Cons. As $G$ is not provable, Cons is not provable either.

Let's pause a moment on the extraordinary pun that has been constructed. Let's write, for short, " $(P A, A) \vdash B$ " to say that $B$ is a consequence of the axioms of $P A$ with the additional hypothesis A. So (PA,Cons) designates the axioms of $P A$ to which we have added the formal consistency hypothesis, Cons. Let's now observe that $P A \vdash($ Cons $\rightarrow G)$ and $(P A, C o n s) \vdash G$ are equivalent (it is an obvious result of propositional calculus). We also use the abbreviation $P A \nvdash B$ to state that $P A$ does not prove $B$. We can synthetically rewrite the first and second theorems (the left-right implication of the second), respectively:

If $P A$ is consistent, $P A \nvdash G$ and $P A \nvdash \neg G$.

$$
(P A, \text { Cons }) \vdash G \text {. }
$$

The passing from point (5) to point (6) is most important and is seldom highlighted. Under the meta-theoretical hypothesis of consistency, point (5) says that PA does not prove $G$ nor its negation. On the other hand, if we formalize consistency in the theory, by Cons, and if we add it to $P A$ as a hypothesis, we can then formally deduce $G$ in $P A$ (!). In both cases, be it an issue of proving the undecidability of $G$ or of proving $G$, the hypothesis of consistency is essential and gives different results. More precisely, after having encoded the meta-theory within the theory, by means of Gödelization and by the construction of Theor (points (1) and (2)), now points (5) and (6) prove that the theory is in a way "stronger". Indeed, with the hypothesis

\footnotetext{
${ }^{9}$ There has been some debate on the actual meaning of Cons: does "Cons" really expresses consistency? (Piazza and Pulcini, 2016) rigorously confirm the soundness of the approach we informally followed here and clarify the issue by a close proof-theoretic analysis.
} 
of consistency, encoded and added, $P A$ does prove an assertion which is formally unprovable if we suppose consistency only at the meta-theoretical level ${ }^{10}$.

This is a definitive stop blow to Hilbert's vision. As Weyl and Wittgenstein thought, Meta-Mathematics, when rigorous, is part of Mathematics. Once again, Gödel proves this with points (1) and (2): Theor encodes in theory $P A$ the metatheoretical character of demonstrability. Using points (5) and (6) he also shows that the theory is even more expressive than the meta-theory (or, as we will see better in 5.1, the meta-theoretical deduction of $G$ from consistency follows from the deduction in the theory). In particular, a finitistic meta-induction does not exist: it is a form of induction, which can be perfectly encoded by theoretical induction. The use of the terms meta-theoretical or meta-mathematical can be practical, namely from a didactic standpoint, for instance to distinguish between the "consistency of $P A$ " and Cons. But it is not "fundamental": one cannot found Mathematics, nor any other form of knowledge, by having recourse to its own meta-knowledge, which still has a mathematical (or that knowledge's) form, as Wittgenstein had observed

there is no game which is a meta-game: it is yet another game.

No meta-language can found language:

we are locked in the prison-house of language,

also wrote Wittgenstein. As for Arithmetic and its mathematical extensions, Gödel's coding locks up the prison by a powerful circularity.

As a philosophical conclusion beyond Wittgenstein, let's observe that only a "genealogy of concepts", said Riemann - that we must entrench, with language of course, but beyond language, before language, beneath language, in action in space (Poincaré), such as ordering, or in time (Brouwer: the discrete flow of time) can propose an epistemological analysis of Mathematics, as meaningfull knowledge construction within the world, between us and the world, to organize and understand the world. Both space and time active experiences are needed in order to propose the conceptual invariant, in language, the notion of integer number, which then becomes independent from each one of these actions, yet preserves its structural, geometric meaning. Mathematics is grounded in the "primary gestures" of knowledge, such as pursuing or tracing a trajectory, drawing borders and then posing "lines with no thickness" (Euclid's definition beta), (Longo, 2005, 2016). Language and writing stabilize geometric practices in space and time by the intersubjectivity of our human communicating communitee, (Husserl, 1933). Thus, the philosophy of mathematics should not be just an annex of a philosophy of language, as it has been "From Frege to Gödel" (the title of a famous book) and till now, but a component of a philosophy of nature and its sciences, see (Weyl, 1949) and (Bailly and Longo, 2011).

10 (Piazza and Pulcini, 2016) prove the truth of Cons in the natural or standard model of $P A$, by applying Herbrand's notion of "prototype proof" - a proof of a "for all" statement, by using a "generic" element of the intended domain, instead of induction. This is a key notion also for the analysis of true and interesting (non-diagonal, like $G$ ) but unprovable propositions of $P A$, see below and (Longo, 2011). Formal induction is not the bottom line of the foundation of mathematics, even not for the (meta-)theory of Arithmetic. 


\subsection{And What About "truth"?}

Easy and customary popularizations of Gödel's theorems continue to be proposed — including by illustrious colleagues, in terms of Platonizing "ontologism", often to impress the reader by ontological miracles in Mathematics. Such popularizations still adhere to the rapid and ontologically naïve reading of Gödel's theorem. There are many who invoke, making big gestures and gazing towards the sky, the stupefying existence of true but non-provable assertions, such as $G$ (an ontological or quantum miracle: and what if the understanding of $G$ 's unprovable truth was due to quantum processes in the brain?). In the face of such assertions, one must always ask how can we state that $G$ is "true"? Besides, how can we state, in Mathematics, that an assertion is true without demonstrating it (or taking it for hypothesis)?

The interlocutor must then produce a proof convincing us of the "(unprovable) truth" of $G$. The hypothesis of consistency, he/she points out, implies that $G$ is unprovable (first theorem). And since $G$ "asserts" that it is not provable (it is equivalent in $P A$ to $\neg$ Theor $(G)$ ), then it is true. This reasoning based on the "meaning" of $G$ is informal, vague and unwritten, as we observed. But once formalized, it is a semantic version of the rigorous formal implication $P A \vdash($ Cons $\rightarrow G)$ that constitutes the core of the second theorem. As a matter of fact, the latter formally deduces $G$ from Cons, and therefore proves $G$, once Cons is assumed. So, once we give ourselves an interpretation of $P A$ in the model of standard integers (which gives consistency, that is the truth of Cons), $G$ is "evidently" true, because it is a provable consequence of Cons. Ultimately, we prove the truth of $G$, and any Platonist will also be forced to do so, at least by handwaving. And we do prove it, even easily, in $P A$ and from Cons: that is the second theorem. As we were saying, we return to the extraordinary finesse of Gödel's result, to the subtle interplay between points (5) and (6). And there is no need for a miracle of ontology or of quantum effects, but just classical logic and a reference to the second theorem when trying the unneeded excercise of interpreting the first - a perfect formal diamond, independently of any interpretation.

We will later mention the "concrete" results of incompleteness, that is, combinatorial assertions of Number Theory (of the type "for any $x$ there exists $y \ldots$ and a complicated numeric expression in $x$ and $y$ "), which are interesting and which are not provable in $P A$ - not even from Cons - but only by infinitary extensions of induction or by "prototype proofs" (see the previous note for references). Given their combinatorial complexity, no one dares say of these that they are "evidently" true by invoking ontological or quantum miracles. We are reduced to demonstrating them, of course outside of $P A$, as we will explain ${ }^{11}$. What is Gödel's responsibility in this?

\footnotetext{
${ }^{11}$ In the ontological search for an unprovable mathematical truth, sometimes the "fact" that $G$ must either be true or false is used. Or — this amounting to the same thing — that either $G$ or $\neg G$ must be true, without saying which because it would be necessary to prove it. This "weak ontology of truth" comes from a classical and legitimate hypothesis (the excluded middle) but one which is very strong and semantically unsatisfactory (or highly distasteful - and this is important in Mathematics) when it is question of discussing the truth of assertion $G$ : this is a formal rewriting of the liar paradox which is precisely neither true nor false. Gödel also uses the excluded middle
} 
The 1931 article is perfect: there is not a single assertion, not a single proof, nor a single argument which calls to "truth" or which refers to an interpretation of the formal game. Only in the introduction does Gödel want to informally explain the meaning of the First Theorem and he notes that $G$, the statement which will be unprovable, is sound. But he immediately adds that the specific analysis of the meta-theoretical reasoning which proves it — and which we outlined - will lead to "surprising results" from the standpoint of the "proofs of consistency of formal systems" (the Second Theorem).

Of course, the ontological vision can still be salvaged: the proof is only an access to a pre-existing reality which may sometimes be more than only formal. More specifically, we can give a good notion of relative truth to the relationship between a formal system and a given mathematical structure. For example, imagine the sequence of integers with the properties learned in elementary school. You know how to say that $4+3=7$ is true, or that $667 \times 52=34084$ is false, or that $7<8 \ldots$ The formal theory $(P A)$ makes it possible to demonstrate it automatically (and a machine does this far better than we do). It is though possible to consider these properties as "true" or "false" by associating to the signs of the theory the concrete and meaningful numbers from one's school-age experience. In general, we will say that a formal theory is "sound" if it proves only true assertions in the associated (or standard) model and, following Hilbert, that it is "complete" if it proves all true assertions in this model.

Alfred Tarski indeed proposed in the 1930s a general theory of truth (Tarskian Semantics), the foundation of the new and very relevant logico-mathematical Theory of Models. It associates to each formal sign the corresponding "object" in the associated structure (the model): " 0 ", as a sign, corresponds to the first element of the well ordered structure of integers; the function sign Succ will be the passage to the next one, following the order etc.. The formal description adapts to the underlying structure and, from it, everything will be derived. Developing a general theory of the truth of the linguistic and scientific expression as "adaequatio intellectus et rei" is a very delicate endeavor. The misuses, inspired by the works of Tarski with no refernce to its technical depth, were numerous. Some extended Tarskian semantics for example to historical languages and observed, say, that "snow is white" is true when snow is white (brilliant!). So "grass is green" is almost always true, whereas "grass is blue" is almost always false. Yet, this would have been a difficult observation for the Ancient Greeks, who had only a single word for designating both colors green and blue. And we would have trouble refereeing a dispute between two Eskimos where one would be saying "today, the snow is white5" and the other would be saying "the snow is white7" (it seems that Eskimos have over 20 different names for designating the whiteness of snow). Color is not a precise and defined wavelength, but a human act tracing delimitations within a quasi-continuum of wavelengths, an act that is rich in intersubjectivity and history. And the whole construction of objectivity goes likewise. But for Arithmetics, in a first approximation, such semantics may suffice and the reader can be satisfied with what he or she understood at school:

( $G$ is independent from classical $P A$ ) but precisely to give us, in Proof Theory, the "middle": the undecidable. 
one needs only to associate the formal signs to the elementary-school comprehension of integers. But we will see how the notion of truth (or of "element of reality" as Einstein will say), becomes an enormous challenge in Quantum Mechanics; we will return to this while examining its alleged "incompleteness".

As for now, we can summarize Gödel's proof of the existence of a statement - and he actually constructs one - that is true in Tarski's sense and that is not formally provable. We could explain that it is true because PA proves it from the formal hypothesis of consistency: that is, if we suppose that Cons is true, since $P A \vdash($ Cons $\rightarrow G)$ and $P A$ is sound, $G$ is also true ${ }^{12}$.

The historical importance of Gödel's article must now be clear, not only regarding the foundations of Mathematics, but also for the techniques invented throughout the First Theorem's proof. Gödelization and the definition of the class of recursive functions will pave the way for the Theory of Computability and hence for the works of Church, Kleene and Turing during the 1930s. These thinkers, especially Turing, will in turn establish the foundations of modern Computer Science by starting off with - and we insist on this point - entirely logico-mathematical problems: the question of undecidability and the definition of the computable real numbers (that is, those that are effectively generated by means of an algorithm). It is interesting to note how Gödel and Turing (five years later) invented the rigorous notion of computability or of effective decidability within the framework of formal languages and systems. By this, they also definitely stabilized the notion of Hilbertian formal system (Poincaré's "sausage machine"). Yet, they aimed to demonstrate that it is possible to exhibit undecidable propositions and uncomputable processes (which can not be automatically generated, like sausages, without using stronger hypotheses). To say no, it is necessary to define exactly that to which one says no. And then, if it is interesting, it can be made even more usable, ultimately taking the form of the digital machine (an arithmetic or Turing machine) which is in the process of changing our world.

As with Poincaré's three-body theorem, the negative result is the starting point of a new science due to its content and to the methods it proposes. It must be noted that in 1931, the scope of the analysis of computability proposed by Gödel was not obvious. Gödel, who was aware of this, wrote in the end of his article that his result did not necessarily contradict Hilbert's formalist point of view. One coud possibly find other formalizations for the informal notion of effective deduction which would not necessarily be encodable using his recursive functions. It is only with the equivalence results of all formal systems for computability proved by Turing and Kleene in 1935 and 1937 that we will have a proof of the generality of Gödel's method.

Church's thesis will propose the mathematical invariance of all notions of computability and of the notion of effective deduction or of acceptable deduction as

\footnotetext{
${ }^{12}$ Let's recall for the reader who may be somewhat numbed by this wonderful pun that the question resides in the difference between the meta-theoretical hypothesis of consistency and Cons, the theoretical hypothesis of consistency which encodes consistency in $P A$. When this extra assumption is added to $P A$, then, we insist, Gödel could formally derive $G$, within $P A$, thus its truth in the standard model, which realizes Cons, if $P A$ is consistent (see the work in (Piazza and Pulcini, 2016) and the previous notes as for the meaning of Cons).
} 
regards finitism. And in a 1963 note, Gödel will recognize the full generality of his theorem: it bases itself on a "sure, precise and adapted" notion of formal system and contradicts the decidability, completeness and (formally) provable consistency "of any consistent formal system which contains a sufficiently expressive finitary theory of numbers". And the search for extensions that are specific to (and consistent with) formal Arithmetics and Set Theory will mark the developments of Logic during the following decades ${ }^{13}$.

\section{Poincaré vs. Gödel}

We have attempted to explain how Poincaré's Three-Body Theorem can be seen as a "philosophical precedent" for Gödel's theorem. Unpredictability resembles undecidability, in time and space - in a sense, statements on future space configurations are undecidable. From a philosophical point of view, Poincaré always appreciated unsolvable problems, "negative results". But technically, both theorems can not be directly correlated; be it only because Laplacian predictability is a problem of the interface between the mathematical system and the physical process and not a purely mathematical question as is Hilbertian decidability. We can, however, establish a mathematical correlation between certain consequences of these two great theorems. We just give some hints here to an analysis more closely surveyed in (Calude and Longo, 2016).

Poincaré's geometry of dynamical systems extends physico-mathematical determination and captures randomness, contrarily to Laplace's distinction. Classical randomness, as we have said, is unpredictable determinism - a fundamental insight by Poincaré to be recalled, as too often randomness is still opposed to determinism. Now, this randomness can also be given by purely mathematical means without reference to physical processes. Birkhoff provided a definition of it in the 1930s, following one of his important results. In very informal terms, if we give ourselves an observable in a particular dynamic (the speed or momentum in each point, for example), a point is said to be random if the average of the temporal evolution of the observable for the point coincides at infinity with the average of the observable on the full space (the temporal average coincides asymptotically with the spatial average). Think of a particle in an isolated volume of an ideal gas: its average speed over time will be equal to the average speed of all of the particles making up the gas. If we push this asymptotic analysis of an average to the actual limit, as the coincidence of two integrals, one integral expressing the average over time the other

\footnotetext{
${ }^{13}$ We can mention Gentzen's ordinal analysis (1935). Larger infinities, as orders beyond integers or as cardinals beyond the countable, provide tools for the analysis of proof in order to fill the incompleteness of $P A-$ or to postpone it to stronger theories. Set Theory, with an axiom of infinity, in its formal version (ZF or NBG) extends and proves consistency of $P A$, but it does not prove its own consistency - it is incomplete, of course, nor is able to answer the questions for which it was created: the validity of the axiom of choice and of the continuum hypothesis. The respective independence results cast additional light on the expressivity and on the limits of formal systems (Kunnen, 1980).
} 
over space, we obtain a mathematical means of defining a random movement, and even a random point — the origin of the trajectory, (Petersen, 1983).

Let's return to Gödel. In the 1960s, P. Martin-Löf proposed a notion of randomness for infinite sequences of numbers (for example of $0 \mathrm{~s}$ and $1 \mathrm{~s}$ ) that is based on Gödel's (in-)computability. The idea, proposed in a doctoral thesis directed in part by Kolmogorov, was then further developed by G. Chaitin, (Calude, 2002). In short, the notion of "effective statistical test" is defined in terms of computable functions; informally, the possible regularities or computable segments in a sequence are effectively checked. A random sequence must not have any effectively recognizable regularity which is repeated indefinitely. Then all possible effective tests are enumerated and an infinite sequence which passes "all effective tests" is qualified as $(M L)$-random (for Martin-Löf random): that is, randomness for an infinite sequence is defined by the property of "passing all effective tests for regularities" or no "regularity" can be effectively detected. Note that this asymptotic construction is necessary to deal with randomness in full generality. Kolmogorof had conjectured that incompressiblity for finite strings could caracterize randomness. Martin-Löf showed that any infinite sequences possesses finite compressible initial segments. Even more strongly, any sufficiently long finite sequence is compressible, by Van der Waarden theorem, see (Calude and Longo, 2016a).

It is easy to prove that an (ML)-random sequence is strongly undecidable in Gödel's sense: it is not only undecidable and even impossible to effectively generate (it is not semi-decidable), but, especially, no infinite sub-sequence of it can be effectively generated (it contains no infinite recursively enumerable sub-sequence). The interesting fact here is that asymptotic dynamic randomness, a la Birkhoff, and the "Gödelian" ML-randomness are equivalent. And, indeed, if one gives a structure of effectivity (effective metric spaces etc) to a vast and interesting class of physicomathematical dynamics, from weakly chaotic (mixing) dynamics to full chaoticity, one can demonstrate the coincidence of Poincaré-Birkhoff randomness and MartinLöf gödelian randomness, see (Gacs et al., 2009) or (Calude and Longo, 2016) for a survey.

Let's be clear, Poincaré's theorem cannot be deduced from Gödel's (nor can the opposite be done). However, as we have said, the approaches proposed by the one and the other, and more specifically by reinforcing their negative results, allow to give purely mathematical limit notions of dynamical or algorithmic randomness. And these notions may be brougth to coincide. Let's observe that the introduction of classical randomness in deterministic systems, i.e. considering it as unpredictable determination, is a very important element of the new vision of dynamic systems proposed by Poincaré. In the same way, undecidability is at the center of Gödel's theorem - and algorithmic randomness is a (strong) extension of it.

Let's finally note that to prove the equivalence, asymptotically, of algorithmic randomness and of the randomness of physical dynamics does not signify at all that the "the Universe is a (great?) algorithm". To the contrary, we have demonstrated that in a deterministic framework, asymptotically and under certain hypotheses, dynamic randomness or unpredictability coincides with algorithmic randomness, which is a (strong) form of undecidability. So, by contraposing (the Contrap 
rule stated above) of this equivalence, an algorithmic procedure, a method of semidecision or a computable (recursive) function only generates predictable deterministic processes. Now, not only the dice of course, but also the solar system (or just three celestial bodies) and almost all which surrounds us is a fabric of correlations and, therefore, forms a "system". This makes most physical processes better described by are non-linear mathematical systems, as interactions yield non-linearity. In view also of physical measurement, which is always an interval, and to sensitivity to initial conditions, they are therefore unpredictable and, a fortiori, non-computable. In this frame, measurement by interval, that is this thinking in terms of interval or "natural" topologies over continuous mathematical structures, is crucial.

In summary, the comprehension of the world provided by continuous Mathematics and the one provided by discrete Mathematics differ: the world is not the sum of little squares or of little points, as are Seurat's paintings, whose access (measurement) is exact. As soon one deals with a dynamics, not just the approximation of a static image by pixels, continuous and discrete space trajectories differ, approximation becomes a major challenge. This has been discussed by many authors, its dramatic consequences in the understanding of biology are hinted in (Longo, 2018).

However, randomness, as unpredictability in the intended physical theory, may be brought to coincide, asymptotically, with algorithmic randomness, a theory grounded on discrete data types: "negative results" in a sense "converge" at the infinite limit. This is at the root of very interesting further work relating algorithmic randomness both to classical dynamics, as mentioned above, and to statistical physics (thus thermodynamics), see (Baez and Stay, 2012) for example. It may be so anytime randomness and limit processes play a role in the intelligibility of physical phenomena. Also Turing, during the last few years of his short life, dealt with continuous vs. discrete dynamics, (Turing, 1952), the fundamental aporia of Mathematics, as observed by Réné Thom.

\subsection{Turing: From Formal Systems to Continuous Dynamics}

"The Discrete State Machine", wrote Turing in 1950 concerning the Logic Machine he invented in 1936, the prototype of the digital computer, "is Laplacian": unpredictability can only be in practice (due to a long and complicated program), and does not exist in principle, he insists, as it does in the physics of "continuous systems". Thus he defines the systems he will study in his fundamental article of 1952 dedicated to morphogenesis (the continuous dynamics of forms). In his 1952 nonlinear continuous systems of action/reaction/diffusion equations, the evolution of forms - for instance color patterns on an animal's fur — is sensitive to the initial conditions: it is subject to "exponential drifts" or to "catastrophic instability", says Turing. Imperceptible changes, over time, to physical measurement and therefore to any discretization, can cause great differences over time. Turing completely shifts his area of research and perspective. 
He discusses the problem and works in the wake of Poincaré, all the while limiting the analysis of solutions to linear approximations - as he focuses at length on the the non-linear case. Continuous dynamics replace his first Machine's sequence of discrete states. The computation is no longer based on a fundamental distinction, which he invented, between software and hardware (which are both discrete), but is rather a continuum of deformations, a continuous genesis of forms, of only the physical. Turing's morphogenesis is a purely hardware/material dynamics of forms.

Let's very briefly attempt to grasp the meaning of Turing's reflection. Due to this change in point of view, we will understand why the correlation result between dynamic randomness and algorithmic randomness contributes in turn to the formal negation of the myth of a universe completely accessible to numericals computations. By approximation, these computations transfer equational determinations to discrete data bases; here the access to the data is exact, contrarily to physical measurement which is always an interval. Moreover, due to successive rounding-off, the orbits of chaotic dynamics, when they are computed by a machine, quickly differ from the physical orbits described in continuous space-time. So the sensitivity to initial conditions can be hidden in a theory of algorithms, one which is necessarily arithmetic, and discretization imposes evolutions which are different than those we are able to describe in the mathematical continuum.

Take the best computer simulation of the double pendulum (it's easy, there are only two equations; such simulations can be found on the Internet). If you launch the pendulum once and again using the same initial values, the algorithm will cause the simulated pendulum to take the exact same trajectory, be it one thousand or ten thousand times. But this does not make any physical sense. Indeed, the unpredictability of the (random) evolution of the actual physical device is very simple to show and is precisely characterized by the fact that, launched again using the same initial conditions (in the physical world where measurement is not exact and is by nature an interval), it generally does not follow the same trajectory. Due to the sensitivity to the initial conditions, after a few oscillations and from the very interval of the best possible physical measurement, it follows different orbits. Continuous Mathematics tell us this a priori. And some call "random" a physical process precisely when, repeated under the "same" initial conditions (in the physical sense), it does not follow the same evolution.

This is foreign to Algorithm Theory, and it is only artificially that one who has understood can imitate the physical unpredictable dynamics. One can, for instance, add to the time of each new launch, a one-number shift to the left or to the right according to a random number taken from the Internet (for instance, is there an odd or an even number of people using Skype at this very moment?). But this is an "imitation" and not a "modelization" of the physical phenomenon. In this respect, Turing makes a very subtle distinction between imitation (the game described in the 1950 article) and model (1952). The latter does not seek to deceive the observer, as does imitation but rather to make intelligible the examined physical process (morphogenesis) and to propose a structure of determination for it, the equations. For example, the sensitivity of the double pendulum to fluctuations in temperature is not made intelligible, from the point of view of "causality", by the recourse to randomness taken 
from the network in a discrete state machine. It is just (but effectively) imitated. The differential equations of its movement, a mathematical model, provide on the other hand its formal determination; they make it intelligible, by highlighting the forces at play and enable to analyze the divergence of trajectories (the "exponential drift", says Turing, the so called Lyapounov exponents). Turing elegantly contributed to the debate by teaching us, both as for discrete state machines and, later, for continuous dynamics (morphogenesis), how "to be within phenomena", (Longo, 2018L).

We are not saying that the world is continuous rather than discrete. It is what it is. We are only saying that Continuous Mathematics, since Newton, enables to understand the properties of physical dynamics which elude Discrete Mathematics. The unavoidable interval of classical physical measurement, with the possible fluctuations/perturbations within, is better grasped by continuity. In a theory of the numerical, below the proposed discretization, nothing else can happen in principle, but also in actual applications: the repetition works - woe if there lacked a comma in a file that has been opened a thousand times! Nevertheless, Discrete Mathematics, in turn, once implemented in extremely powerful machines, allows to analyze processes, chaotic ones in particular, that mathematical conceptual analysis can absolutely not reveal. Hence they provide us also with another type of intelligibility, one which is just as important.

In short, from the physical standpoint, a theory of algorithms does not produce an accurate model of the whole world, but of a small set of deterministic systems: predictable systems. And once transferred to the realm of the discrete, all deterministic systems become predictable, even if they are the implementation of non-linear equations or functions. It is possible to perfectly repeat, against Physics, even the wildest of turbulences. And it is not true that the discrete is an approximation of the continuous. Numerical analysts very well know that difficult "shadowing" theorems (cf. (Pilyugin, 1999)) are required to prove that, in the numerical implementation of certain chaotic dynamics, the continuous trajectories "shadow" the discrete ones (and not the opposite). In general, the discrete is not an approximation of the continuous. It is, at best, the opposite: a given discrete trajectory can be approximated by a continuous one. So the images, displayed on a computer screen, of a chaotic evolution give qualitatively important information regarding continuous trajectories: they provide very useful imitations that are now indispensable for science and its applications. And the richness of science and technology, the variety in history, from Lorenz onwards and especially since the 1970s, is so that we appreciate chaos on the screens of digital machines more than anywhere else. The meteorologist can look at turbulence and hurricane simulations over and over, and can repeat them identically if desired. He/she can thus have a better grasp on what appears to be the most interesting aspects and, based on experience, can make increasingly reliable predictions.

In a very specific sense then, any algorithmic theory of the physical universe is mathematically "incomplete" with respect to continuous descriptions. And the aforementioned theorems, which link classical and algorithmic randomness, demonstrate it again, by duality (or by contraposition, as we said). If Gödel's theorem sets limits to any attempt at a mechanical reduction of mathematical deduction, its con- 
sequences (we will see other ones) also obliterate the algorithmic visions of an inert universe - and let's not even mention the living state of matter, the brain for example - because, as mentioned above, there are problems even in the algorithmic simulation of the double pendulum: no program follows the physical dynamics. And when limits are better understood, it becomes possible to use our tools at their best and improve them, from Proof Theory to digital simulation; the latter being science's main instrument today.

As concerns the continuous/discrete dichotomy, even within Theory of Computation, the proofs of abstract properties of discrete structures (see (Kreisel, 1984) for a discussion and references) or the analysis of today's computer networks may require a difficult use of geometric tools, in the continuous realm. The latter are indeed immersed in a relativistic space-time, which we better understand using continuity (cf. for example (Goulbault, 2000) for a relevant use of homotopy theory in Concurrency Theory in computer networks). As for the discreteness of Quantum Mechanics - which some could invoke as an ultimate discretization of the world

- the phenomena of entanglement or of non-separability are at the opposite of the topological separations specific to discrete databases in which each point is isolated, well-separated from all others. As a matter of fact, let's examine this further possible meaning of "incompleteness".

\section{Einstein and the Thesis of the Incompleteness of Quantum Mechanics}

Einstein was certainly no stranger to the debate concerning the foundations of Mathematics, firstly through his active collaboration in Zurich with Weyl who published a book in 1918 on the foundations of Mathematics (The Continuum) as well as another one, a veritable mainstay, on the mathematical foundations of Relativity (Space, Time, Matter). Einstein would later meet again with Weyl in Germany, as well as with Hilbert. He will also witness from afar the foundational contention between Hilbert and Brouwer, the founding father of intuitionism, a dispute that will result in the exclusion of Brouwer from the editorial board of the very prestigious mathematical journal directed by Hilbert. Such a preposterous outcome will be rather appalling to Einstein (he would call it a "batrachomyomachia" referring to ancient Greek comedy).

Einstein also had the opportunity to discuss with Von Neumann, also in exile from Nazi Germany at the Institute for Advanced Studies, where Weyl will also move to in 1933. Von Neumann had a good knowledge of Gödel's theorem. It is even said that when Gödel, at the age of 24 , presented his result before a meeting in 1930, Von Neumann was the only person to grasp the scope of it. The result actually had a shattering effect on the staunch formalist who was Von Neumann; he had worked on Hilbert's program, as did Ackermann and so many others, and at a point he had been briefly convinced of having obtained an acceptable proof of the consistency of $P A$. The great mathematician was rather swift: after having 
heard the first theorem, he drew from it the purely formal proof of the second one. However, by the time he informed Gödel of this, the latter's article was already under print with the two theorems. Thus, not only did Von Neumann know Gödel's theorems, but he had even worked on them. He then presented them to Princeton mathematicians and physicists at one of his first seminars during the fall of 1931 (Sieg, 1994). Later, Gödel himself would also temporarily move to the Institute, in 1933-1934 ${ }^{14}$. During the following years, Von Neumann developed his hyperformalist approach in several fields ranging from the axiomatization of Quantum Mechanics to Probability Calculus and Game Theory, formal games of economics and war.

In 1935, Einstein will write an article with Podolski and Rosen that will be known under the initials "EPR" in which they will examine the problem of the "sound" and "complete" character of Quantum Mechanics (QM). These terms are specific to Mathematical Logic (we have used them) and are not common in Physics, especially in what concerns the term "completeness". It is therefore more than likely that it is no coincidence that the authors used the term "completeness" to criticize the descriptions of physical reality proposed by quantum formalism: they most probably imagined they would be dealing another blow like Gödel's against Hilbert. The Gödelian paradigm will in any case serve as a tool for comprehension: almost surely so for them, most definitely so for us. We use the term "paradox" when referring to EPR, as it is often done, thus reminding of the employment of proof by contradiction as used by Gödel, as well as emphasizing the "paradoxical" aspect of QM (it is indeed a theory which is often positioned against the classical "doxa", in physics).

EPR begins by stating with great clarity the ontological hypotheses of the whole reflection: even in microphysics, there must exist a physical reality that is independent of measurement and of theory. At most, the measurement can "disturb" the measured physical quantity. As regards the theory, it must, of course, be sound : a "satisfactory" theory must only lead to true assertions. Then, in order for it to be complete, "every element of the physical reality must have a counterpart in the physical theory", meaning that it must be described or deduced within the theory. One will recognize a requirement of "semantic" soundness and completeness, as we mentioned concerning Logic, as well as an ontological reading of these properties.

The classical semantic interpretation of Gödel's theorem tells us precisely that, in hypothesis of consistency of $P A$, the assertion $G$ is valid over the natural numbers (of course, we observed, since Cons implies $G$ ), but that the formal theory, $P A$, is not enable to deduce it. EPR seeks a complete theory regarding a physical reality whose objects of knowledge, even in microphysics, must unambigously be accessible (well-separated in space) by measurement and separated as well from the knowing subject. And it demonstrates, under this ontological hypothesis, that current QM does not constitute one. The arguments used by EPR are based on various fundamental aspects of QM, among which those we know under the names of "indetermination" and of "entanglement".

\footnotetext{
${ }^{14}$ Gödel moved there permanently in 1939, after a spectacular escape from Nazi-occupied Austria.
} 
Quantum indetermination may be described as the non-commutativity of the measurement of the position and momentum of a particle. According to the theory, the values obtained depend on the order in which these measurements are made and therefore, as it is stated in EPR,

we can no longer speak of the physical quantity $A$ [or $B$ ] having a particular value.

Also,

if the operators corresponding to two physical quantities, say $A$ and $B$, do not commute, that is, if $A B \neq B A$ then the precise knowledge of one of them precludes such a knowledge of the other.

And EPR continues: the two physical quantities of position and momentum therefore "cannot have simultaneous reality" and at least one element of reality will not be described.

If then the wave function provided such a complete description of reality, it would contain these values; these would then be predictable.

As regards entanglement, EPR deduces it from an observation which will become fundamental. From quantum formalism (Schrödinger's equation in particular), it is shown that if two systems interacted at time $t=0$ and then were separated without any further interaction until time $T>0$, it would be possible to know the value of a measurement over one of the systems at time $T$ by performing this measurement on the other system. Two "entangled" particles, as we are saying, allow for an instantaneous knowledge of the value of a measurement made on the one because of the measurement made on the other. If the first has an "up" spin, for example, the result of measurement of the other spin will be "down". By repeating the same process, we can obtain the "down" spin for the first; the other will then have an "up" spin, if measured. Is this an instantaneous propagation of information, one which happens faster than the speed of light? That would be impossible, it would contradict Relativity. The theoretical explanation by QM is either inconsistent or incomplete, says EPR.

To summarize, EPR points out the incompleteness entailed by a fundamental property of the gap between theory and measurement in QM, if consistent: that which is computed, with the wave function (Schrödinger's equation), is not what is measured. In Classical and Relativistic Physics, computations are made over real numbers taken from measurements. These computations, in turn, produce real numbers which are verified by means of other measurements. In QM, computations are made over complex numbers in Hilbert spaces that are very abstract, possibly having an infinite number of dimensions, and which are therefore outside of usual spacetime. Then, real numbers are produced as projections (modules) of complex numbers obtained by means of the computations. These values are the probability of getting certain results in the process of measurement and, when verified by means of measurement, they are, on the one hand, dependent of the order in which the measurements are made (non-commutativity) and, on the other hand, they can be correlated if the particles, which are measured, are entangled (or which are in an "EPR" state, as physicists would still put it today). 
And even recently, "hidden-variable" theories have tried to fill (to complete) these gaps in QM, its incompleteness. However, it is the "standard interpretation" which prevails, emphasizing the originality of the construction of knowledge in QM. Measurement is consubstantial with the physical object: there is not already a particle traveling along with its properties and states "already given" and which is to be, at most, disturbed by the measurement. If we launch a "photon" against a double slit and if we measure, using an interferometer, the result on a wall beyond the slits, we will observe interference, a typical wave-like behavior. If, conversely, we put a particle counter behind each of the slits, we will "observe" a particle passing $50 \%$ of the time on one side and $50 \%$ of the time on the other. The action of measurement, the consequence of a whole theoretical framework, gives the specification of the object. The scientific concept of photon isolates a fragment of the universe which is specified in the theoretical and practical act of its own production and of measurement: a wave or a particle.

Likewise, Schrödinger's equation enables to calculate the evolution of a system of entangled particles and provides "correlated" values of probabilities for eventual measurements. In short, if we throw two classical coins into the air which then interact (for example, if they collide), and then take their own distinct trajectory without any further interaction, the two probabilistic analyses of the heads or tails values taken by the two coins will be independent. On the other hand, the Bell equations (Bell, 1964) and the Aspect experiments (Aspect et al., 1982) demonstrated that the measurements (probability values) for two entangled quanta (having interacted) are correlated, not independent. If we know the one, we know the other, even at a great distance; this confirmed EPR's theoretical deduction. No "information" passes between the two distant events: it is necessary to make, a posteriori, a phone call in order to verify that the two measurements are indeed entangled.

This fact, undoubtedly extraordinary ("paradoxical") and now empirically verified several times over, is at the origin of very interesting theoretical reflections of which the practical consequences could be significant: Quantum Computing. Such a "calculus" could revolutionize actual computing: in the very least, computations that are impossible to perform because they are too complex would become quite feasible because entanglement is a form (a very original one) of "parallel computing". But what is being computed? It is not numerical information as we usually understand it, but the evolution of a system, which is global: the two particles are not separable by measurement and a variable associated to the object would not be local (it would not depend on the evolution of a "single point"). These are absurdities, from the standpoint of classical and relativistic physics, which EPR deduces from the theory and which have been verified empirically. As we were saying, the world is not made up of little dots or of little squares, of classical bits and bytes that are well separable by the unique way we have of accessing them: the active constitution of scientific objectivity and of objects of knowledge, in the friction between ourselves and the world, which is measurement (sense or instrument-based).

Let's finally note that we have not said here that $\mathrm{QM}$ is complete, but that the proof given by EPR of its incompleteness is neither theoretically valid nor empirically corroborated: entanglement is there, it does not contradict physical evidence. 
EPR argument for incompleteness is founded upon topological (and ontological) hypotheses, the well separated locality of measured observables, that are inadequate as regards microphysics. EPR thus declared the impossibility of a situation that has been empirically shown to be possible (and very interesting). Einstein was wrong, but when he observed that

QM is incomplete because entanglement is deduced from its theoretical and mathematical structure,

he first paved the way for research and experiments, and then for possible machines which may become of great importance ${ }^{15}$.

\section{The Concrete/Mathematical Incompleteness of Formal Theories}

Following Gödel's theorem, the opposition between various schools of thought regarding the foundations of Mathematics deepened. Federigo Enriques said so with great lucidity in Paris, 1935:

[...] if we avoid the Scylla of ontologism, we fall into the Charybdis of nominalism: could an empty and tautological system of signs satisfy our scientific reason? From both sides, I see the emergence of the ghost of a new scholastic (Enriques, 1983).

One the one hand, the invocation of the eternal and pre-existing "truth", certain because absolute, that

the mathematician discovers by looking over God's shoulder (John D. Barrow).

On the other hand, the insistence on the mathematical certitude founded upon the absence of ambiguities of meaning, on the mechanical nature of deduction and, why not, of all reasoning. Then some claimed that our humanity, could be fully transferred to a Logico-Mathematical machine, eventually producing the so-called super-brains foreseen by Artificial Intelligence in the 1960s and 1970s. Indeed, the formalists (nominalists) will say for many years, Gödel's theorem demonstrates the independence of a meaningless diagonal assertion. It is an astute, rather farfetched paradox; it is of no importance as regards interesting mathematical deduction and even less in what concerns human reasoning.

On the contrary, Gödel's theorem is only the starting point of an avalanche of formally unprovable assertions, among which some are very interesting. They are assertions of Formal Number Theory with a mathematical sense and of mathematical interest and which can only be demonstrated by means of more powerful arguments

\footnotetext{
${ }^{15}$ Deduction in EPR may remind of another, from Aristotle:

the void is impossible, because in it, all objects would fall at the same speed (La Physique, vol. 4, chap. 8).
}

Great theoretical minds, even when they are mistaken, propose very interesting ideas indeed. 
than those provided by formal finitism. To pass from one line of such arguments to the next, it is necessary at some point to invoke such a thing as "meaning" or "infinity". Let's try to explain this non-obvious matter, very briefly (more may be found in (Longo, 2011)).

To remain within the sphere of Logic, let's recall that Gentzen (1935) gave a proof of the coherence of Arithmetics by using transfinite induction; a result which will inaugurate modern Proof Theory, in the form of "Ordinal Analysis". In short, he demonstrated the consistency of $P A$ by transfinite induction over a restricted class of formulas (roughly: induction with an infinity of hypotheses, reaching the ordinal $\varepsilon_{0}$, an infinity which is "small", but which is large enough to resolve the equation $x=\omega^{x}$, where $\omega$ is the infinity of integers). The restriction to a certain type of formula and the rigor of proof, in an original framework called "natural deduction", will make the proof convincing, but it is obviously not formalizable in $P A$. In 1958, Gödel himself will give a proof in a "stratified" system (numbers, then functions over numbers, etc. $\ldots$ the typed $\lambda$-calculus). Let's note that this proof will be extended, in a non-trivial manner, by Girard (1970) to a system based on second-order quantification, that is, on "for all ..." or "there exists" also over sets or types (PA is a first order theory: only number variables are quantified). Girard's system turned out to be mathematically challenging and highly successful in Computer Science, for introducing a strong form of modularity in programming (Girard et al., 1990), (Asperti and Longo, 1991). Of course, here also, the effectivity of the calculus cohabits with the formal unprovability of its consistency, of which the proof is only formalizable in third-order Arithmetics (sets of sets) and which implies the consistency of $P A$. Thus, while with Gentzen begins the use of larger and larger ordinals in order to give infinitary proofs of the consistency of increasingly expressive theories; with Gödel or Girard, we pass onto higher orders, as quantification over infinite sets or types.

So, in order to salvage the paradigmatic theory of the finite, $P A$, it is necessary to have recourse to forms of infinity; in Mathematics, infinity is a diffcult but omnipresent concept. We need only to think about the birth of infinitesimal calculus and the associated notions of instantaneous speed and acceleration, indispensable to Physics after Newton and obtained as limits to the infinity of finite approximations. Or to Projective Geometry, born in $X V^{\text {th }}$ century Italian painting, and in particular in the Annunciations, where a symbolic form of divine infinity, the vanishing point at the back of the painting, made the space on the finite plane more human. In Mathematics, the infinite helps to better understand, describe, organize the finite. And Set Theory demonstrates this: to formalize the concept of "finite", it is necessary to have an axiom of the existence of infinity (in $P A$, it is impossible to formally isolate standard, finite numbers, and hence to define the "finite"). Whether finitist formalists like it or not, the mathematical concepts of finite and of infinite are formally "entangled", inseparable: if we wish to formally capture the finite, it is necessary to work with the concept of infinity.

In 1978, Paris and Harrington published a combinatorial assertion, PH say, also inspired by Logic, but one which was not artificial, a rather "meaningful" mathematical statement (Gödel's $G$ is not so, according to many), formalizable in $P A$ and with- 
out any apparent relationship to consistency (formalized by Cons $\equiv \neg$ Theor $(0=1)$ in section 5). From this statement, it is possible to deduce Cons in $P A$, so $\mathrm{PH}$ is therefore unprovable. But it is possible to prove it, outside of $P A$, with Gentzen-like transfinite induction. We will mention another result, one which is similar but even more interesting. As a matter of fact, both proofs are similar and what we are about to say applies, implicitly, to the proof of Paris-Harrington's statement.

In a 1981 unpublished note, H. Friedman gave a finite version, formalizable in Arithmetics, of a famous theorem on finite trees. The trees, which in Mathematics, grow from the top towards the bottom of the page or the blackboard, are familiar and useful structures, with numerous applications. In particular, Kruskal's theorem (Kruskal, 1960), which Friedman "miniaturized", proves a property which is widely used, especially in Mathematical Computer Science (for halting problems in formal systems of calculus, or of "rewriting", (Bezem et al., 20003)). We informally hint here to a result that has been discussed in several books and papers, see (Harrington et al., 1985), (Gallier, 1991) among others. An analysis of its (un-)provability is also in (Longo, 2011).

It is easy to imagine how to say that a tree is included in another, that is to give a partial order between trees. Then the theorem says that no infinite sequence of trees can be completely disordered, i.e. there always exist comparable trees, the first included in the second, in the order - thus there are no infinite decreasing sub-sequences - and this has very interesting applications also in computing (term rewriting).

Friedman's Finite Form $(F F F)$ "renders in the finite" the infinitary statement of Kruskal (which concerns infinite sequences of finite trees). $F F F$, for any $n$, gives the length $m$ of the finite sequence in which we find two comparable trees. $F F F$ is formalizable in $P A$ : it is a "for any $n$ there exists an $m$ such as ( ... )" statement, where " (... )" is a property which is encodable in PA (finite trees are easily Gödelizable) and which is decidable (once $n$ and $m$ are fixed). Now, the function which associates $n$ to $m$ is computable, but it increases so fast that it definitively majorates any recursive function provably total in $P A$ (and also in strong extensions of it). This is a way to prove the unprovability of $F F F$ in $P A$.

Friedman, for his earlier proof, immerses trees in transfinite ordinals and, thanks to the absence of infinite decreasing sequences (that is, by transfinite induction), demonstrates that FFF implies Cons in PA. And so, by this very difficult tour de force, he demonstrates that $F F F$ is formally unprovable, a consequence of Gödel's Second Incompleteness Theorem (Cons is unprovable). With one or the other technique, the proof of unprovability constitutes a surprising logical and mathematical feat to which a whole book was devoted shortly following the dissemination of the 1981 note, (Harrington et al., 1985).

The observation to be made is that many of the applications of Kruskal's theorem are also obtainable from Friedman's arithmetic form. It is therefore clearly something other than an artificial/logical trick: it is Mathematics. And yet $F F F$, as well as its negation, are formally unprovable: this is the reason why we have called this section The Concrete/Mathematical Incompleteness of Formalisms, something we would have been unable to do if we were only thinking of Gödel's "logico- 
antinomical" statement $G$ which is not very "mathematical" - nor conretely talks of numbers nor trees of/orders on numbers.

Now, in order to demonstrate the undemonstrability of $F F F$ 's negation, we can only prove something stronger: that $F F F$ is true for integer numbers (or in any model of $P A$ ). There is no way of kidding here and claim that its truth is God given or due to quantum effects in the brain - all remarks based on a superficial reading of Gödel's theorems - with no reference to its actual proof. One only has to prove the statement. It is indeed Mathematics, not just Logic. So how is it possible to show that $F F F$ is true (holds) in this structure? Of course, we cannot make a finite formal induction, an induction in $P A$, due to its undemonstrability. The proofs given by Friedman and in the book we mentioned use induction in a way that is quite usual for mathematicians who do not work on foundations.

\subsection{Towards the Cognitive Foundations of Induction}

To explain and possibly justify such a use of induction, we will adopt a strong epistemological position, one which develops Riemann's reference to the foundations of Mathematics as a "genealogy of concepts", Poincaré's reflections on the role of action in space for the constitution of mathematical concepts, those of Enriques sometimes vague, but often very stimulating - on the various forms of sensorial access to space, and the unity of Weyl's thought regarding symmetries as principles of conceptual construction, in Mathematics and in Physics. These great geometers, opposed to formalism, opened up, in a very incomplete and informal manner, avenues for foundational reflections of a strictly epistemological nature. They are sometimes revisited today in terms that are cognitive, relatively general and scientific, and beyond introspection, which was the only means of investigation at the time. We refer to the 1997 books by Berthoz and Dehaene and to previous reflections by this author, (Longo, 2005).

It is then possible to understand the incompleteness of formalisms as an insufficiency of the "principles of proofs" (of which informal induction is the paradigm) for capturing the "principles of construction" (firstly, well-ordering and symmetries) - the latter are increasingly shared with theoretical construction in Physics, whereas their principles of proof differ, cf. (Bailly and Longo, 2011).

The mathematician says and writes the following every day: if a set of integers regardless of how it is defined - is non-empty, then it has a smallest element. You, the reader, see (we hope) the sequence of integers, well-ordered from left to right (for those of us who write in this direction, for those who write Arabic, it is the opposite). Look at it carefully, in your mind, as an infinite sequence of well separated numbers growing towards the horizon rather than on paper: 1, 2, 3, 4 .. If we isolate in the sequence, conceptually, an ordered set of integers numbers containing at least one element, we may observe that this set contains a smallest element - at worst it will be 0 : the set is discrete and without infinite decreasing sequences - technically, such a set is said to be well-ordered. This is a common practice in numerical intu- 
ition, one which is prohibited to the formalist because it is geometric and because it evokes "meaning", meaning as the act of counting or ordering. It is an act that is rich in signification - of writing, of ordering in space, of making this repeated movement towards the horizon. It originates in a human gesture (or maybe even in the pre-human one, in what concerns small numbers (Dehaene, 1997)), of ordering (small) countable quantities together. It also refers to the "sense" of the discrete flow of time, in Brouwer's approach. Meaning is thus rooted in ancient gestures that are, in that, extremely strong. Language and writing gave them the objectivity of intersubjectivity, the stability of common notation, and independence as regards the objects denumerated. The number and its order are first practical then conceptual invariants that make sense thanks to the independence they acquired with respect to a plurality of uses and acts of life, in space and in time. By repetition in space, by means of language and writing, we construct this discrete and increasing sequence to which the mathematician easily applies the abstract principle of "well order" thanks to its rich geometric meaning: a non-empty set of integers has a smallest element.

The mathematician uses such a signifying structure, one which evokes order in space and time, everyday and even also to construct a formal axiomatic, as did Peano and Hilbert, as a last stage in the construction of invariance or independence. But this last step, formalization, does not enable to completely separate the proof and its theory from meaning, of space and time and in space and time, which is constituted in this genealogy of concepts which is behind all of mathematical construction ${ }^{16}$. This is what the mathematical incompleteness of formal systems means: the principles of (formal) proof do not have the expressivity of principles of construction (order in space or time and symmetries) having produced the conceptual structures of Mathematics - they are incomplete w.r.to our active, concrete and meaningful mathematical structuring of the world ${ }^{17}$.

It is thus that even mathematicians who philosophically support or who are close to formalism demonstrate, in the 1985 book onwards, the validity of Friedman's statement by invoking, in a repeated but highly visible way, the principle of "wellorder". With calm certitude, they pass at some point from the argument of one line to the next by observing that a non-empty set of integers, defined in the demonstration, has a smallest element. Such proof is perfectly rigorous and is founded upon a most solid cognitive practice: the invariance and conceptual stability of well-order specific to the "gestalt", rich in meaning, of the sequence of integers. Contrarily to so many formalizations, it does not entail contradictions. This is how the formally unprovable proof works.

Of course, some mathematicians later produced a detailed analysis of the proof, since it is not formalizable in $P A$. They demonstrated that the well-ordered set eludes

\footnotetext{
16 (Husserl, 1933):
}

Original certainty can not be confused with the certainty of axioms, because axioms are already the result of the formation of meaning and always have such formation of meaning as a backdrop.

${ }^{17}$ For technical details regarding order and symmetries in the demonstrations we refer to, see (Longo, 2011). 
finitist formalization, since it implicitly uses infinite quantification (over sets, $\Sigma_{1}^{1}$ technically, cf. (Rathjen et al., 1993)). They thus prove Friedman's statement using induction over a huge transfinite ordinal, one which is far greater than that proposed by Gentzen and which is definable by means of a very diffcult construction. Some justify the infinitary audacity by observing that the set involved in the well-ordering statement is non-empty only by hypothesis of a "reductio ad absurdum". It will then disappear precisely because it gives rise to absurdity ... it will therefore be empty. And yet, this detour by infinity is necessary, because of the proof that the assertion is unprovable in a finitary way (its unprovability in $P A$ ).

But has then all of this work been useless? Even the Greeks could believe in the consistency of Arithmetics, they who "saw" the potentially infinite and well-ordered sequence of integers with, scattered in their midst, the prime numbers. Gödel's theorem is a pun with no "mathematical" meaning; the mathematical statements which do have meaning are demonstrated using presumed infinite sets which are not definable in $P A$, sets that, in the end, turn out to be empty ... Is then this detour useless? Not at all, this path is extremely rich, in itself and because of its spin-offs. Simply, in what concerns the play between the finitude and infinitude of numbers, of space, it traverses all Mathematics. It began with the use of potential infinity with Euclid, as apeiron (limitless). Then, it was clarified by Aristotle, and refined by the Thomist school, which was used to working with the diffcult and controversial infinity of the Christian God: thanks to its contribution, we clearly established the distinction between potential and actual infinity, specific to God. Then came projective geometry, as we were saying, a first mathematical consequence of the practice of actual infinity, followed by infinitesimal calculus, both having entailed huge developments and applications.

It was then necessary to clarify how demonstrations were made, particularly when using this limit concept and in particular following the brilliant congestion of XIX th century Mathematics; how rigorous definitions are produced, after a century that was so prolific mathematically, although its mathematics often lacked rigor. The formal systems turned out to be incomplete, but far from useless: they taught us how to produce good definitions, how to rigorously generalize, how to unify methods and proofs using the axiomatic method ... . The mistake was rather to think that it was possible to work without meaning in order to prove consistently, mechanically, rigorously; to be able to avoid any reference to action in space and in time, which are the loci of the constitution of Mathematics, even that of integers. However, as we have mentioned, it was necessary - in order to demonstrate that there are undecidable statements - to specify what is meant by decidable or computable in a mechanically certain way; and so were set the Mathematical bases, with Gödel and Turing, of Computer Science. And in the end, we are brought back, but with a whole set of tools, to this sense of space and of action within, to its

[...] geometry, generated in our human space from a human activity (Husserl, 1933) ${ }^{18}$.

\footnotetext{
18 The date at which Husserl's manuscript was written reminds us that almost all of the story we have told took place during the first and dramatic half of the $X X^{\text {th }}$ century, 1933 being a pivotal year, with the rise of Nazism and the flight from Germany of so many people we have met in
} 


\section{Information and Encoding in the Cell}

In calling the structure of the chromosome fibers a code-script we mean that the allpenetrating mind, once conceived by Laplace, [...] could tell from their structure whether the egg would develop, under suitable conditions, into a black cock or into a speckled hen, into a fly or a maize plant $[\ldots]$

wrote Schrödinger in his 1944 book during his exile in Ireland from Germany. The immense figure of Laplace remains in the backdrop of the whole history we have examined, beginning with Poincaré's work. Some of the thinkers we mentioned here explicitly recognized his mark upon their own scientific analysis: in sect. 6.1, we quoted Turing's remarks on the "laplacian" nature of his Discrete States Machine.

Schrödinger proposed in 1944 the idea of seeing, halfway between metaphor and science, chromosomes as a "code-script", encoding hereditary information. And from his perspective as a physicist, he understood its implicitly Laplacian nature and he provided prudent and plausible examples. In the second part of the book, though, Schrödinger hints

[...] to the possible meaning of the principle of entropy at the global scale of a living organism, while forgetting for the time being all what we know on chromosomes.

In particular, Shrödinger investigates the possible role of "negative entropy" as a form of Gibbs' free energy (available energy for work). He opened by this yet another possible path for reflection in organismal biology (see (Bailly and Longo, 2008) and (Longo and Montévil, 2014, chap. 9) for more in this direction hinted by Schrödinger).

What can we find in common between these various forms of determination which involve predictability and therefore a full understanding of the world based on a few equations and a few signs? The expressive completeness of writing, more specifically of alphabetic writing, can provide a key for interpreting the omnipresence of this way we have of doing science.

The Laplace equations are of course formal or formalizable writing which were believed, up until Poincaré, to be a complete determination, i.e. able to predict the

these pages. During that year, Husserl, who was 74 years old at the time, was prohibited from publishing and even from accessing the University Library. And this frequent appearance of some illustrious names reminds of another important/small academic/political story. In 1923, Einstein, having recently been awarded the Nobel prize, thought about returning to Italy, maybe for a long period, after a short stay in Bologna. He had a very good knowledge of the results by Levi-Civita and was in contact with several colleagues, among whom Volterra and Enriques. The latter, in the previous years had become familiar in dealing with the governments, managed to obtain a meeting with the new prime minister, formally not yet dictator, Benito Mussolini: he hoped to obtain exceptional financing for the guest. This was in early 1924. The Duce's response was : "Italy has no need for foreign geniuses" - this reminds by contraposition of the great Princes of the Renaissance or of Princeton in the 1930s (and afterwards). And so Einstein did not return to Italy. In 1929, Marconi added to a list of his colleagues drafted for Mussolini a little e. (for Jew - "ebreo" in Italian) in front of the names of the three aforementioned Italian mathematicians, the greatest of their time. The Duce, nine years prior to his racial laws, excluded them from the Academy of Italy (Faracovi et al., 1998). 
possible evolutions of the physical universe - with beside it randomness that was supposed to be distinct from equational determination. We have also recalled in the first section how, for Laplace, the fundamental level is always in the elementary, in the simple particles of which it is necessary to isolate, describe, and then integrate the movement into systems by the progressive sum of individual behaviors.

Hilbert, in turn, will make explicit the discrete nature of mathematical formalisms, as a sequence of simple and elementary material, alphabetic signs: sequences of signs, the axioms, were meant to completely allow to deduce, "determine", the properties of the intended mathematical structures. He paved the way for Turing's digital machine, once letters and words had been Gödelized - encoded by numbers. Alpha-numeric formal systems should have told us everything about them. And for some, Turing's machine should have fully modeled at a point the functioning of the brain. If it remains each time audacious, the process of knowledge seems to increasingly narrow itself and deteriorate. It is original and justified in the case of Laplace and Hilbert and two immense theorems were required, by Poincaré and Gödel, to undo them - theorems which were made possible by the mathematical rigor of the original proposals. But this project hits rock bottom when reaching the 0 and 1 of a brain seen as a digital switchboard, in Classical Artificial Intelligence, or when reaching the four-letter alphabet of the nucleotide bases which compose DNA. The latter becomes

the program of any individual's behavioral computer (Mayr, 1961)

(Mayr later opposed the idea of a central role for genes in evolution). And the assumption of completeness assures that the

DNA contains all information required for the reproduction of the cell,

and of the whole organism (Crick, 1966).

So the

one gene - one enzyme (Beadle et al., 1941)

hypothesis and then the

Central Dogma of molecular biology

(information passes linearly and unidirectionally from DNA to RNA, to proteins and then to the structure of the organism (Crick, 1958)) are of a Laplacian nature as regards the structure of determination they suggest: DNA is complete, it contains the information for any phenotype and information propagates in a linear fashion and in a single direction from it ("one gene - one enzyme" and the "dogma"). The first hypothesis was considered to be valid for over fifty years before it was demonstrated to be false; as it concerns the dogma, it still permeates research in molecular biology (in cancer etiology, for example, see below), although it has recently been rejected by the majority, albeit not always aloud.

More or less implicitly the idea of completeness of DNA w.r. to all phenotypes is still prevailing, in spite of growing empirical evidence against those claims and various alternative proposals for organismal biology — some work is synthetised 
in (Soto et al., 2016). Note, though, that the complete knowledge of the chemical structure of the DNA and the alleged identification of all human "genes", its "decoding", has been a major technological success, in 2001. Unfortunately, the number of genes keeps changing, from about 80,000 in 1999, still assumed by the head of the Human Genome Project launched in 1990, (Collins, 1999), to 25,000 in 2001, down to about 20,000 today ${ }^{19}$.

It is not our aim here to develop such considerations further, see for example (Fox Keller, 2000). Our goal is to compare the scientific practices, as for negative results, in physics and mathematics to those in a relatively young and very important field such as Molecular Biology. As for an analysis of the incompleteness of genocentric analyses in biology, let's first note that the hypotheses or dogmas which were at the basis of numerous works for such a long time, and which purported to be "physicalistic" or "materialistic", seem to not have taken enough into account what happened in physics, (Longo and Tendero, 2007), (Longo, 2018). Since Poincaré, we have understood that in the presence of simple interactions (only three celestial bodies), the initial measurable situation does not contain all "the information" (to use a rather unfitting expression) on future trajectories, if we mean by that the "complete determination" of the system's evolution. And we remain Laplacian when adding to what is "necessary" a fragment of randomness, as "noise", quite distinct from the former, as done in (Monod, 1970) - recall that Poincaré had integrated the two. Monod's necessity, because of its Laplacian nature, turns out to be programmable (the theory of the "genetic program").

What seems to be neglected, in the hypotheses and dogmas regarding sequential molecular cascades, is that even Physics of the $X X^{\text {th }}$ century, after Relativity, sees the universe as a fabric of interactions: if the interactions change, the fabric and its space are deformed; if we act upon the fabric and upon space, the interactions change. The Central Dogma is foreign to this vision of interactions as constitutive of a unity specific even to contemporary physics; and it concerns, let's recall, objects within a structure such as the cell and the organism, where almost everything is correlated to almost everything. Noise as well is a largely inadequate notion to understand the role of randomness as biological unpredictability, see (Bravi and Longo, 2015), as well as of stochasticity in genes' expression, (Elowitz et al., 2002). In general, the focus on discrete structures sets a bias on the analysis of determination and randomness, see (Longo, 2018).

DNA is, of course, a most important component of the cell, but the analyses of life phenomena, which base themselves solely upon it and upon the molecular cascades that follow, are incomplete, in a sense which is indeed impossible to specify within a theorem but which is suggested by Physics itself. When we see that it is described as "the book in which the essence of life is written", we realize that the alphabetic myth still governs a part of science: it is a myth in the Greek sense, a positive myth which is a powerful constructor of knowledge, but which needs to be continuously reviewed and to have its own limits brought to light. From Dem-

\footnotetext{
${ }^{19}$ In 1999, Collins was pleased to stress the difference between humans and Caenorhabditis Elegans, a one millimeter worm with less than 1,000 cells, whose DNA had just been decoded: it has only 19,000 genes!
} 
ocritus - who fragmented the world into atoms and who associated them to the letters of the alphabet - to Descartes - for whom certitude is obtained by decomposing reasoning into elementary and simple components - , and to Laplace and Hilbert, certainty in understanding must always refer to the elementary and simple, to the atomic and alphabetic. The model of alphabetic reconstruction, discrete and elementary, of the continuous song of language has been presiding our sciences for millennia, with extraordinary productivity: we wish to understand everything in this manner. So, we have believed for centuries that, like language with the alphabetic structure, we can reconstruct, for all of knowledge, the world by projecting letters onto it and that these completely determine the intended structures, in Physics, in Logic, in Biology (atoms, the sequences of signs of a formalism, the letters of DNA). In other words, discrete signs and letters make it possible to express all which is sayable and therefore all which is thinkable ${ }^{20}$ So, from Mathematics, to Physics and Biology, the signs and discrete sequences of signs (formal encodings) contain the full determination of all possible evolutions, at all levels of phenomena.

Now, it is necessary to highlight the strength and limits of this vision of knowledge, its incompleteness to put it shortly. Indeed, even the image of the language thus proposed, as an instrument of human communication, is quite incomplete. We forget that the "compiler" or "interpreter" of alphabetic languages is the production of sound, by a composition of phonemes: meaning is in the spoken-sung and in its expressivity. It is necessary to read, to produce a sound - even silently in one's head - to find meaning, in the same way that a musician hears music, "interprets it", when reading a score, which is another form of alphabetical writing of the musical continuum, (but a two-dimensional writing which is enriched with symbols and signs of continuity). So the context, sometimes linguistic and written, and the tone, the gesture or the drawing contribute in an essential way to expression and to comprehension ... in sum, to meaning. Furthermore, a pout, a smile, a punch, making love, all of these enable to say something else, and contribute to human expressivity, to what is thinkable, in a essential way, beyond and with the sequences of alphabetic signs. In the same way, the meaning in space of the well-order of integers is part of mathematical proof and, for the fervent anti-formalists we are, of its foundations, in the epistemological sense, with but beyond formal systems, demonstrated to be incomplete.

So, to return to Biology, we are slowly steering away from the alphabetic myth, which is unfortunately still the priority in what concerns financing, and which claims

$\overline{20}$ (F. Jacob, Leçon inaugurale, Coll. France, 7 mai 1965):

The surprise is that genetic specificity is written not with ideograms, like in Chinese, but with an alphabet.

In this perspective, see (Jacob, 1974) for more, also the philosophy of biology, reduced to Molecular Biology, is transformed in an annex of a philosophy of (alphabetic) language, cf. sect. 5 above. As a matter of fact, Molecular Biology deals with information, programs, expressions, signals ... since "life is fully coded" in chromosomes, following (Schrödinger, 1944), thus in discrete sequences of meaningless signs, as theorized also by (Maynard-Smith, 1999), (Gouyon, 2002) and many others. 
that the stability and organization of DNA, and of molecular cascades stemming from it, fully determine the stability and organization of the cell and of the organism. This myth is false, because the physical and biological stability and organization of the cell and of the organism contribute causally to the stability and to the organization of DNA and of the molecular cascades which follow from it. Isn't this circular? We are used to such challenges: let's recall what Gödel did with a very subtle circularity, far away from logicist fears. The problem of "how it started", the origin of life, remains in any case enormous. With no membrane, without a cell, an organisms, no significant metabolic cycle is created, even less is it maintained over time. Similarly to what Gödelian incompleteness led us to understand, for Mathematics with respect to formal systems, "strict" extensions (in the logical sense) of molecular theories seem necessary in order to say something more about the physical singularity of the living state of matter, see (Bailly and Longo, 2011) and, for recent advances on an organismal perspective, (Soto et al., 2016) ${ }^{21}$.

Let's conclude these considerations with some questions and by identifying general challenges. Why should the fundamental always be the "elementary"? Galileo's theories of gravitation and inertia, which are undamental theories, tell us nothing about Democritus's atoms which did however constitute his masses. Einstein unified inertia and gravitation; he proposed another theory, the relativistic field, also fundamental, without saying anything about quanta. Of course, the problem of unification with the quantum field is an issue. However, physicists will say unification, and not reduction: it is a question of putting fundamental theories into perspective, of modifying them both in view of a synthesis to invent. The greatest progresses are possibly achieved today by reconstructing, from quantum measurement, the geometry of space and time, see A. Connes's "non-commutative geometry".

And finally, why should the elementary always be simple, as if we were transposing the alphabetic and Cartesian method to the phenomena at hand? Two frontiers of contemporary knowledge, microphysics and the analysis of life phenomena, seem to call for another vision: their elementary components, the quantas and the cell (which is elementary, atomic, since it is no longer alive if we split it in two) are very complex. Their comprehension requires approaches that are "non-local", to use quantum terminology, or systemic analyses, as many increasingly put it in Biology (see (Soto et al., 2016) for work and references), well beyond the supposed causal completeness of DNA and well beyond the myths, from Mathematics and Physics to the analyses of human cognition, of the completeness of alphabetic formalisms.

\section{Appendix: on Gödelitis in biology}

In an attempt to bypass the mechanistic-formal approach, enriched by some noise, (Danchin, 2003, 2009) tried to bring Gödel's theorem into the genocentric view of

\footnotetext{
21 The empirical evidence on the incompleteness of the genocentric approach as for a dramatic phenotype, cancer, and some general consequences on the understanding of causality, in particular on the etiology of that disease, are discussed in (Longo, 2018).
} 
biology. Within the formal-linguistic approach to biology, Gödel's incompleteness would prove the "creativity" of biological dynamics by recursion and diagonalizing on the programs for life: in short, the DNA would generate unpredictable novelty by a creative encoding of phenotypes, a la Gödel. A remarkable attempt for a leading biologist, as these issues in Logic are far from common sense, as we hinted above. Indeed, (Rogers, 1967), a classic in Computability Theory, calls "creative" the set of (encoded) theorems of arithmetic, i.e. the formal-mechanical consequences of its axioms. As we know, by Gödel's first theorem, this set is not computable (not decidable) - and, to the biologist, its evocative name may recall Bergson's Creative Evolution. However, this set is semi-computable (semi-decidable), meaning that it may be effectively generated and, as such, is far from "unpredictable", since an algorithm produces all and exactly all its infinite elements - the set of encoded theorems. Moreover, the generation of Gödel's undecidable formula is effective as well: it is an incredibly smart recursive and "diagonal" construction (it recursively uses the encoding of logical negation), as we have seen, which allows to construct a formula not derivable from the axioms. This procedure may be indefinitely and effectively iterated.

In short, Gödel's undecidabile sentence is effectively produced by an effective encoding of the metatheory into the formal theory and it does not finitely "create" any "unpredictable "proposition : the diagonal formula may be constructed ,even though it is not derivable from the axioms. In summary, on one side, formal deriv ability is not decidability (Godel 's first theorem ), as the axioms do not allow to decide all formulae, typically Godel's diagonal formula. Yet they stillyield semi-computability or semi-decidability: the theorems can be effectively generated, by passing through the encoded metatheory (what would be the metatheory in evolutionary biology?). On the other side, the construction of the sentence that escapes the given axioms is also effective (semi-computable), as wehave seen.

Theoretical unpredictability, instead, that is the least property one expects for "creativity" in nature, is at least (algorithmic) randomness, for infinite sequences (sect. 6). This yields a very strong form of incomputability, far from semi-computability. As observed in sect. 6, a random set of numbers and its complement cannot even contain an infinite semi-computable subset. This form of randomness may be soundly compared, asymptotically, to unpredictability in physics, as we observed (note that biological unpredictability includes both classical and quantum randomness, (Buiatti and Longo, 2013), (Calude and Longo, 2016)). We also observed that finite incompressibility does not soundly relate to randomness in nature: an incompressible sequence may be programmable - by a program of its length or just one bit longer; moreover, there are no sufficiently long incompressible sequences, (Calude and Longo, 2016a) - except by a restriction on the allowed machines, a la Chaitin (Calude, 2002). In summary, physical/biological randomness is unpredictability relative to the intended theory, (Calude and Longo, 2016), and a time related issue: it concerns the future and is associated to time irreversibility, (Longo and Montévil, 2014: chap. 7). It relates only asymptotically to algorithmic randomness; it is necessary, but insufficient, for analysing evolutionary changes. It goes well beyond Gödel's constructive diagonal craftiness. 
The merit of Danchin's remarks, though, is that they are based on precise mathematical notions, thus they may be proved to be wrong. This is in contrast to the commonsensical abuses of vague notions of information and program, as mostly used in Molecular Biology, from which strong consequences have been too often derived, see (Longo, 2018).

\section{References}

1. Aspect, A., Grangier, P., \& Roger, G. (1982). Experimental Realization of the EinsteinPodolsky-Rosen-Bohm Gedankenexperiment: A New Violation of Bell's Inequalities. Phys. Rev. Let., 49, 91-94.

2. Asperti, A., \& Longo, G. (1991). Categories, Types and Structures: An Introduction to Category Theory for the Working Computer Scientist. Cambridge, MA: The MIT Press.

3. Beadle, G. W., \& Tatum, E. L. (1941). Genetic Control of Developmental Reactions. American Nauturalist, 75, 107-116.

4. Baez, J., \& Stay, M. (2012). Algorithmic Thermodynamics. Mathematical Structures in Comp. Sci., 22 (5), 771-787.

5. Bailly, F., \& Longo, G. (2009). Biological Organization and Anti-Entropy. J. of Biological Systems, 17 (1), 63-96.

6. Bailly, F., \& Longo, G. (2011). Mathematics and Natural Sciences. The Physical Singularity of Life. London: Imperial College Press. (original version in French, Hermann, Paris, 2006).

7. Barrow-Green, J. (1997). Poincaré and the Three-Body Problem. Providence/London: American Mathematical Society/London Mathematical Society.

8. Bell, J. S. (1964). On the Einstein-Podolsky-Rosen Paradox. Physics, 1 (3),195-200.

9. Béguin, F. (2006). Le mémoire de Poincaré pour le prix du roi Oscar. In E. Charpentier, E. Ghys, and A. Lesne (Eds.) (2006). L'héritage scientifique de Poincaré. Paris: Belin.

10. Berthoz, A. (1997). Le sens du mouvement. Paris: Odile Jacob. (English transl.: The Brain Sens of Movement, Harvard University Press, 2000).

11. Bottazzini, U. (1999). Poincaré. Le Scienze.

12. Bravi, B., \& Longo, G. (2015). The Unconventionality of Nature: Biology, from Noise to Functional Randomness. In C. S. Calude, and M. J. Dinneen (Eds.), Unconventional Computation and Natural Computation (pp. 3-34). LNCS 9252, Springer.

13. Buiatti, M. (2000). Lo stato vivente della materia. Le frontiere della nuova biologia. Torino: UTET.

14. Buiatti, M., \& Longo, G. (2013). Randomness and Multi-level Interactions in Biology. Theory in Biosciences, 132 (3), 139-158.

15. Calude, C. (2002). Information and randomness (2nd edition). Berlin: Springer-Verlag.

16. Calude, C., \& Longo G. (2016). Classical, Quantum and Biological Randomness as Relative Unpredictability. Invited Paper, special issue of Natural Computing, 15 (2), 263-278, Springer.

17. Calude, C., \& Longo G. (2016a). The Deluge of Spurious Correlations in Big Data. Found. of Science, (March), 1-18.

18. Charpentier, E., Ghys, E., \& Lesne, A. (Eds.) (2006). L’héritage scientifique de Poincaré. Paris: Belin.

19. Crick, F. H. C. (1958). Central Dogma of Molecular Biology. Nature, 227 (8), 561-563.

20. Crick, F. H. C. (1966). Of Molecules and Man. Seattle, USA: Washington University Press.

21. Danchin, A. (2003). The Delphic Boat. What genomes tell us. Harvard University Press.

22. Danchin, A. (2009). Bacteria as computers making computers. Microbiology Rev., 33, 3-26.

23. Dehaene, S. (1997). La bosse des Maths. Paris: Odile Jacob. (English transl., Oxford University Press, 1998). 
24. MSCS Editorial Board (2009). Editors note: bibliometrics and the curators of orthodoxy. Math. Struct. in Comp. Science, 19 (1), 1-4.

25. Einstein, A., Podolsky, B., \& Rosen, N. (1935). Can Quantum-Mechanical Description of Physical Reality Be Considered Complete? Phys. Rev., 47 (10), 777-780.

26. Elowitz, M. B., Levine, A. J., Siggia, E., \& Swain, P. S. (2002). Stochastic Gene Expression in a Single Cell. Science, 297 (5584), 1183-1186.

27. Enriques, F. (1983). "Filosofia scientifica", La filosofia scientifica a congresso, Parigi, 1935, a cura di G. Polizzi, in Dimensioni VII, 50.

28. Faracovi, O., Speranza, F., \& Enriques, F. (1998). Filosofia e storia del pensiero scientifico. Livorno: Belforte.

29. Fox Keller, E. (2000). The century of the gene. Cambridge, MA: Harvard University Press.

30. Frege, G. (1884). The Foundations of Arithmetic. Northwestern University Press. (english transl.: Evanston, 1980).

31. Gács, P., Hoyrup, M., \& Rojas, C. (2009). Randomness on Computable Metric Spaces: A dynamical point of view. In 26th International Symposium on Theoretical Aspects of Computer Science, (STACS, 2009) (pp. 469-480).

32. Gallier, J. (1991). What is so special about Kruskal's theorem and the ordinal $\Gamma_{0}$ ?. Annals Pure Appl. Logic, 53, 199-260.

33. Gentzen, G. (1969). The collected papers of Gerard Gentzen, M. E. Szabo (Ed.). Amsterdam: North-Holland.

34. Girard, J. Y., Lafont, Y., \& Taylor, P. (1990). Proofs and Types. Cambridge, MA: Cambridge University Press.

35. Gödel, K. (1986-2003). Collected Works. S. Feferman e al. (Eds.), (5 volumes). Oxford: Claredon Press.

36. Goubault, E. (Ed.) (2000). Geometry in Concurrency: A user's guide. Mathematical Structures in Computer Science, 10 (4), 411-425.

37. Gouyon, P.-H., Henry, J.-P. \& Arnoud, J. (2002). Gene Avatars, The Neo-Darwinian Theory of Evolution. New York: Kluwer Academic Publishers.

38. Harrington, L., et al. (Eds.) (1985). H. Friedman's Research on the Foundations of Mathematics. Amsterdam: North-Holland.

39. Hilbert, D. (1899). Grundlagen der Geometrie. Leipzig: Teubner. (Translated by L. Unger as: Foundations of Geometry, Open Court, La Salle, 1971.)

40. Husserl, E. (1933). The Origin of Geometry. (transl. in English, Univ. Nebraska Press, 1989).

41. Jacob, F. (1974). Le modèle linguistique en biologie. Critique, 30 (322), 197-205.

42. Kunnen, K. (1980). Set Theory: An Introduction to Independence Proofs. North-Holland.

43. Kreisel, G. (1984). Four lettters to G. Longo. http://www.di.ens.fr/users/longo/files/FourLettersKreisel.pdf

44. Kruskal, J. (1960). Well-quasi-ordering and the tree theorem. Trans. Amer. Math. Soc., 95, 210-225.

45. Laskar, J. (1989). A numerical experiment on the chaotic behaviour of the Solar System. Nature, 338, 237-238.

46. Laskar, J. (1990). The chaotic behaviour of the solar system. Icarus, 88, 266-291.

47. Laskar, J. (1994). Large scale chaos in the Solar System. Astron. Astrophys., 287, L9-L12.

48. Lighthill, J. (1986). The recently recognized failure of predictability in Newtonian dynamics. Proc. R. Soc. Lond., A 407, 35-50.

49. Longo, G. (1996). The Lambda-Calculus: connections to higher type Recursion Theory, Proof-Theory, Category Theory. A short (advanced) course on lambda-calculus and its mathematics. Spring 1996. (revision of "On Church's Formal Theory of Functions and Functionals". Annals Pure Appl. Logic, 40 (2), 93-133, 1988).

50. Longo, G. (2005). The Cognitive Foundations of Mathematics: human gestures in proofs. In M. Okada et al. (Eds.), Images and Reasoning, (pp. 105-134). Tokio: Keio University Press.

51. Longo, G. (2009). From exact sciences to life phenomena: following Schroedinger and Turing on Programs, Life and Causality. (Special issue), Information and Computation, 207 (5), 543670. 
52. Longo, G. (2011). Reflections on Concrete Incompleteness. Philosophia Mathematica, 19 (3), 255-280.

53. Longo, G. (2014). Science, Problem Solving and Bibliometrics. In W. Blockmans et al. (Eds.), Use and Abuse of Bibliometrics, Portland Press.

54. Longo, G. (2016). The consequences of Philosophy. Glass-Bead, (web-journal). http://www.glass-bead.org/article/the-consequences-of-philosophy/?lang=enview

55. Longo, G. (2018). Information and Causality: Mathematical Reflections on Cancer Biology. Organisms. Journal of Biological Sciences, 2, 1.

56. Longo, G. (2018d). Complexity, Information and Diversity, in Science and in Democracy. In The Ghost of Transparency: An Architectonics of Communication. Bhlmann, Doyle, Savic (eds), Springer.

57. Longo, G. (2018L). Letter to Alan Turing. in Theory, Culture and Society, Posthumanities Special Issue.

58. Longo, G., \& Montévil, M. (2014). Perspectives on Organisms: Biological Time, Symmetries and Singularities. Berlin: Springer-Verlag.

59. Longo, G., \& Tendero, P.-E. (2007). The differential method and the causal incompleteness of Programming Theory in Molecular Biology. Foundations of Science, 12, 337-366.

60. Maynard-Smith, J. (1999). The idea of Information in Biology. The Quarter Rev. of Biology, 74, 495-400.

61. Mayr, E. (1961). Cause and effect in Biology. Science, 134 (348), 1501-1506.

62. Martin-Löf, P. (1966). The definition of random sequences. Information and Control, 9, 602619.

63. Monod, J. (1970). Le Hasard et la Nécessité. Paris: Éditions du Seuil.

64. Paris, J., \& Harrington, L. (1978). A mathematical incompleteness in Peano Arithmetic. In J. Barwise (Ed.), Handbook of Mathematical Logic (pp. 1133-1142). Amsterdam: NorthHolland.

65. Petersen, K. (1983). Ergodic Theory. Cambridge: Cambridge University Press.

66. Piazza, M., \& Pulcini G. (2016). What's so Special About the Gödel Sentence G? In F. Boccuni and A. Sereni (Eds.), Objectivity, Realism, and Proof: FilMat Studies in the Philosophy of Mathematics (pp. 245-263). Springer.

67. Poincaré, H. (1892). Les Méthodes Nouvelles de la Mécanique Céleste. Paris: GauthierVillars.

68. Poincaré, H. (1902). La Science et l'Hypothèse. Paris: Flammarion.

69. Poincaré, H. (1906). Les mathématiques et la logique. Revue de Métaphysique et de Morale, 14 (3), 294-317.

70. Poincaré, H. (1908). Science et Méthode. Paris: Flammarion.

71. Pilyugin, S. Yu. (1999). Shadowing in Dynamical Systems. Berlin, New York: Springer.

72. Rathjen, M., \& Weiermann, A. (1993). Proof-theoretic investigations on Kruskal's theorem. Annals Pure Appl. Logic, 60, 49-88.

73. Ruelle, D., \& Takens, F. (1971). On the nature of turbulence. Commun. Math. Phys., 20, $167-192$

74. Ruelle, D., \& Takens, F. (1971). Note Concerning our Paper "On the nature of turbulence". Commun. Math. Phys., 23, 343-344.

75. Sieg, W. (1994). Mechanical procedures and mathematical experience. In A. George (Ed.), Mathematics and Mind (71-117). New York: Oxford University Press.

76. Smorynski, C. (1977). The incompleteness theorems. In J. Barwise, and Keisler H. J. (Eds.), Handbook of Mathematical Logic (pp. 821-866). Amsterdam: North-Holland.

77. Soto, A. M., Longo, G., \& Noble, D. (Eds.) (2016). From the century of the genome to the century of the organism: New theoretical approaches. (Special issue), Prog. Biophys. Mol. Biol., 122 (1), 1-82. (doi: 10.1016/j.pbiomolbio.2016.06.006). (Epub 2016 Jul 2. Review. PubMed PMID: 27381480, Oct., 2016).

78. Turing, A. M. (1950). Computing Machinery and Intelligence. Mind, 49 (236), 433-460.

79. Turing, A. M. (1952). The Chemical Basis of Morphogenesis. Philo. Trans. Royal Soc., B 237 (641), 37-72. 
80. Weyl, H. (1949). Philosophy of Mathematics and Natural Sciences, Princeton: Princeton University Press. (original german ed.: 1927).

81. Weyl, H. (1952). Symmetry, Princeton: Princeton University Press.

82. Wittgenstein, L. (1968). Philosophical Remarks, Ascombe ed., University of Chicago Press. 